\title{
DIGITALCOMMONS
}

$@$ WAYNESTATE-

Wayne State University

6-1-2010

\section{Extended Second Welfare Theorem for Nonconvex Economies with Infinite Commodities and Public Goods}

\author{
Aychiluhim Habte \\ Benedict College, Columbia, SC, habtea@benedict.edu \\ Boris S. Mordukhovich \\ Wayne State University, boris@math.wayne.edu
}

\section{Recommended Citation}

Habte, Aychiluhim and Mordukhovich, Boris S., "Extended Second Welfare Theorem for Nonconvex Economies with Infinite Commodities and Public Goods" (2010). Mathematics Research Reports. Paper 77.

http://digitalcommons.wayne.edu/math_reports/77 


\section{EXTENDED SECOND WELFARE THEOREM FOR NONCONVEX ECONOMIES WITH INFINITE COMMODITIES AND PUBLIC GOODS}

AYCHILUHIM HABTE and BORIS S. MORDUKHOVICH

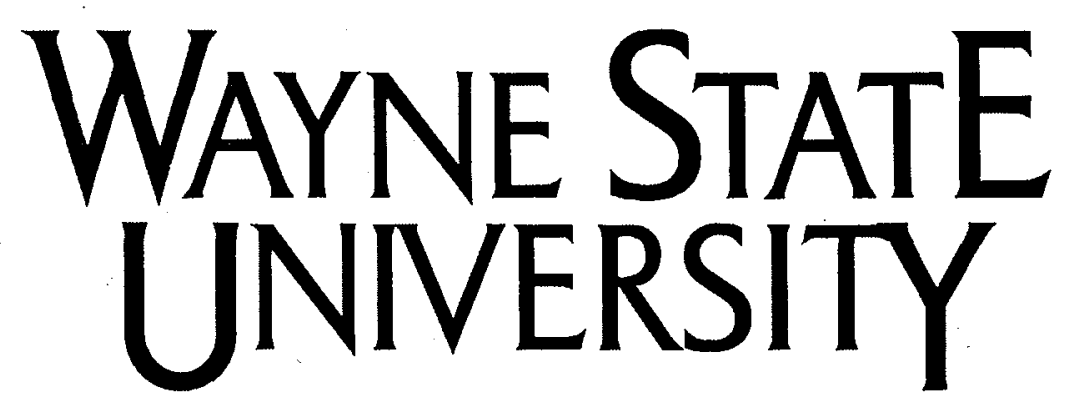

Detroit, MI 48202

Department of Mathematics

Research Report

2010 Series

\#6

This research was partly supported by the US National Science Foundation 


\title{
EXTENDED SECOND WELFARE THEOREM FOR NONCONVEX ECONOMIES WITH INFINITE COMMODITIES AND PUBLIC GOODS
}

\author{
AYCHILUHIM HABTE ${ }^{1}$ and BORIS S. MORDUKHOVICH ${ }^{2}$
}

\begin{abstract}
This paper is devoted to the study of nonconvex models of welfare economics with public goods and infinite-dimensional commodity spaces. Our main attention is paid to new extensions of the fundamental second welfare theorem to the models under consideration. Based on advanced tools of variational analysis and generalized differentiation, we establish appropriate approximate and exact versions of the extended second welfare theorem for Pareto, weak Pareto, and strong Pareto optimal allocations in both marginal price and decentralized price forms.
\end{abstract}

Key words: welfare economics, public goods, Pareto optimal allocations, second welfare theorem, variational analysis, generalized differentiation, extremal principle

JEL classification: D06

Mathematics subject classification (2000): 90A14, 49352

\section{Introduction}

In this paper we consider general nonconvex models of welfare economics involving both private and public goods in infinite-dimensional spaces of commodities. Models of this type have been well recognized from both theoretical and practical viewpoints. Despite a number of excellent works in this area, there are great many of important unsolved problems some of which are addressed in our study.

Recall that in general equilibrium theory a commodity is defined not only by its physical properties but also by the clate, the location, and the state of its nature that precise the condition of its availability. The classical general equilibrium theory deals with a finite mumber of commodities, which implies that the economic activity extends over only finitely many dates, location, and events. Such an assumption, contrary to the framework of many applied models particularly involving location, growth, and finance, dramatically limits the scope of applications of the results obtained to understand the real economic life.

Moreover, the physical property of a commodity can vary continuously depending on some characteristics. In such dynamic settings, a commodity is defined as a point in a space of characteristics describing, e.g., its location, design, quality, functioning, etc. To give a solid foundation to analysis of allocation over time or state of nature was the primary motivation for the study of infinite-dimensional economies; see, e.g., the paper by Debreu and Hildenbrand [11], which was one of the first to seriously address this and related issues.

Our paper concerns the study of infinite-dimensional nonconvex economic models from the viewpoint of Pareto optimality/efficiency. It has been fully recognized that the concept of Pareto optimality and its variants play a crucial role in equilibrium models to make the

\footnotetext{
${ }^{1}$ Dejartment of Mathematics and Computer Science, Benedict College, Columbia, SC 29204, USA; habten.(i)benedict.edu

${ }^{2}$ Department of Mathematics, Wayne State University, Detroit, MI 48202, USA; boris@math.wayne.edu. Research of this author was partly supported by the US National Science Foundation under grant DMS0603846 and by Australian Research Council under grant DP-12092508.
} 
best decisions for competitive economies; see, e.g., $[2,3,9,10,17,20,31]$ with detailed discussions and the references therein.

The classical (foundation) approach to the study of Pareto optimality in models of welfare economics with only private goods and smooth data in finite-dimensional spaces of commodities consists of reducing them to conventional problem of mathematical programming and and the subsequent usage of first-order necessary optimality conditions involving Lagrange multipliers; see Hick [15], Lange [22], and Samuelson [33, 34]. It was shown in this way that the marginal rates of substitution in consumption and production sectors are identical at any Pareto optimal allocation of resources.

The underlying hypothesis in the foundation works on welfare economics $[15,22,33,34]$ was the smoothness/differentiability of the production and utility functions involved in the models. In the beginning of 1950s, Arrow [3] and Debreu [10] made the next crucial step in general equilibrium theory considering models of welfare economics with possibly nonsmooth but convex data in finite-dimensional commodity spaces. Based on the classical separation theorem for convex sets, they established a key result called the second fundamental theorem of welfare economics. This theorem, which is a convex counterpart of the aforementioned result on the marginal rates of substitution, states that any Pareto optimal allocation can be decentralized as price equilibrium, i.e., it can be sustained by a nonzero price at which each consumer minimizes his/her expenditure and each firm maximizes its profit.

The Arrow-Debreu decentralization approach to Pareto optimality has played a profound role in general equilibrium theory and particularly in welfare economics. On the other hand, the relevance of convexity hypotheses is often doubtful in many applications, e.g., for practically realistic models involving the increasing return to scale in the production sector. In fact, it was observed by Samuelson [34, pp. 231-232], even before the appearance the Arrow-Debreu model, that the convexity assumptions are fulfilled "only be accident."

In his pioneering study on price decentralization of Pareto optimal allocations in nonconvex economies with private goods, Guesnerie [14] imposed convexity assumptions not on the initial production and preference sets, but on their tangential approximations via the Dubovitskii-Milyutin cone of interior displacements. Starting with [14], the results of the second welfare theorem type for nonconvex models are interpreted as marginal price equilibria, where marginal prices at Pareto optimal allocations are formalized via the corresponding normal cone that is dual/polar to the tangent cone in question. Guesnerie's approach to nonconvex welfare economics and its elaborations are strongly based on the convexity assumption imposed on the approximating tangent cone.

Further progress in this direction has been achieved by using Clarke's tangent cone in the Guesnerie scheme for various welfare models; see e.g., $[6,9,21]$ and their references. An advantage of Clarke's tangent cone is that it is automatically convex, but a strong disadvantage comes from the fact that the corresponding normal cone happens to be too. large (often the whole space) and thus does not allow us to bring useful information for marginal price equilibria. These issues have been recognized and discussed by Khan [20].

Khan's new approach to marginal price equilibria goes back, in a sense, to the foundation works in welfare economics $[15,22,34]$, which do not use convex separation techniques but reduce the welfare model to an optimization problem that contains at that time nondif- 
ferentiable data. He employs, in the case of finite commodities, the metric approximation method developed by Mordukhovich in nondifferentiable programming [26, 27] and arrived in this way, under appropriate constraint qualifications, at the marginal price equilibrium formalized via the Mordukhovich normal cone, which is much smaller than the Clarke one. Further results in this directions for various models of welfare economics can be found in more recent publications $[4,7,12,16,17,18,23,28,29,31,37]$ and their references.

Concerning economies with public goods, the first fundamental result in the "foundation" direction was obtained by Samuelson [35] who showed that at Pareto optimal allocation the marginal rates of transformation of public goods are equal to the sum of the individual marginal rates of substitution. After more than decade from Samuelson's result, Foley [13] and Milleron [25] established appropriate versions of Arrow-Debreu second welfare theorem for economies with public goods under convexity assumptions. More recent results for nonconvex models of welfare economics involving public goods were obtained in $[19,20,21,36,37]$; see also the references therein.

The main setting of this paper is a general model of nonconvex welfare economics with public goods formulated in the framework of Asplund commodity spaces; see Section 2 for mode details. We develop an approach to the study of this model based on advanced tools of variational analysis and generalized differentiation revolving around the extremal principle; see [30, Chapter 2]. The latter fundamental principle (in its both approximate and exact forms) provides, on one hand, necessary conditions for a certain extremal relationship between closed sets while, on the other hand, can be treated as a variational nonconvex counterpart of the classical separation principle in convex analysis. This approach was suggested in [28] for welfare models with only private goods and then developed in $[4,5$, $23,29,31]$ and other publications. We also refer the reader to $[7,12,16,17,18]$ and the bibliographies therein for similar nonconvex separation ideas closely related to the extremal principle. To some extent, this variational approach via the extremal principle can be viewed as a common roof for the foundation ideas of using first-order optimality conditions as well as for the Arrow-Debreu developments based on convex separation. To the best of our knowledge, such an approach has never been implemented in models of welfare economics with public goods, even in the case of finite commodities.

Developing the variational lines of research, we derive in this paper several new versions of the extended second welfare theorem for nonconvex economies. We start with an approximate/fuzzy version of the second welfare theorem for Pareto and weak Pareto optimal allocations, where marginal prices are formalized via Fréchet/regular normals. Results of this type hold under rather unrestrictive assumptions, but they apply merely to some suboptimal feasible allocations nearby the optimal ones. Imposing additional "normal compactness" requirements on the sets involved in the model, we arrive at an exact/pointwise version of the extended second-welfare theorem with marginal prices formalized via the Mordukhovich normal cone. Furthermore, the usage of the advanced tools of generalized differential and of the associated variational techniques allows us to establish certain decentralized versions of the marginal price results by employing some nonlinear prices. Considering finally, strong Pareto optimal allocations, the notion introduced by Khan [19] and largely underinvestigated in the literature, we obtain the corresponding versions of the extended second welfare 
theorem for nonconvex economies with public goods in the absence of the conventional qualification conditions needed for Pareto and weak Pareto optimal allocations.

The rest of the paper is organized as follows. In Section 2 we describe and discuss a. nonconvex model of welfare economics with both private and public goods in infinitedimensional commodity spaces. We also formulate general qualification conditions employed in the paper for studying Pareto and weak Pareto optimal allocations and present some sufficient conditions for their fulfillment.

Section 3 contains required preliminaries from variational analysis and generalized differentiation needed for deriving the main results of the paper on the extended versions of the second welfare theorem, which are given in the subsequent sections.

In Section 4 we first establish an approximate version of the second welfare theorem with marginal prices formalized via the Fréchet normal cone at Pareto and weak Pareto optimal allocations under the corresponding net demand qualification conditions for nonconvex economies involving public goods. After discussing some remarkable consequences of this result, we derive its decentralized (Arrow-Debreu type) version by using nonlinear prices. This is based on a smooth variational description of of Fréchet normals.

Section 5 is devoted to exact versions of the second welfare theorem for weak Pareto and Pareto optimal allocations under the so-called sequential normal compactness (SNC) conditions imposed on (some of) the sets in question. Conditions of this type are automatic in finite dimensions and always hold for sets with certain Lipschitzian properties in infinitedimensional spaces and can be viewed as far-going extensions of the classical nonempty interior property of convex sets; we do not require the latter even in convex settings.

The final Section 6 concerns welfare economies with ordered commodity spaces. First we establish the price positivity for such models under natural assumptions of the desirability/free disposal type and then derive new results on the fulfillment of an extended version of the second welfare theorem specific for strong Pareto optimal allocations of convex and nonconvex economies with private and public goods.

Our notation is basically standard in variational analysis and economic modeling; cf. $[20,30,31,37]$. Unless otherwise stated, the generic space $X$ under consideration is Banach with the norm $\|\cdot\|$ and the canonical pairing $\langle\cdot, \cdot\rangle$ between $X$ and its topological dual $X^{*}$ endowed with the weak* topology $w^{*}$. Recall that $\mathbb{B}$ and $\mathbb{B}^{*}$ stand for the closed unit ball in $X$ and $X^{*}$, respectively; $\mathbb{N}:=\{1,2, \ldots\}$ is the collection of natural numbers. Given a set-valued mapping $F: X \rightrightarrows X^{*}$, denote by

$$
\begin{aligned}
\operatorname{Limsup}_{x \rightarrow \bar{x}} F(x):=\left\{x^{*} \in X^{*} \mid\right. & \exists \text { sequences } x_{k} \rightarrow \bar{x}, x_{k}^{*} \stackrel{w^{*}}{\rightarrow} x^{*} \text { as } k \rightarrow \infty \\
& \text { with } \left.x_{k}^{*} \in F\left(x_{k}\right) \text { for all } k \in \mathbb{N}\right\}
\end{aligned}
$$

the sequential Painlevé-Kuratowski outer/upper limit of $F$ as $x \rightarrow \bar{x}$. Some more specific symbols are defined in the text below. 


\section{Nonconvex Economies with Private and Public Goods}

Let us first formulate a general and well-recognized by now model of welfare economics with private and public goods; see, e.g., $[20,35]$. This model denoted by $\mathcal{E}$ involves two categories of commodities: private and public. Consumption of the first type is exclusive, i.e., what is taken by one individual automatically becomes unavailable for all the others. In contrast, a good is public if its consumption is identical across all the individuals. Mathematically this means that the commodity space $E$ is represented as the product of two spaces $E=E_{\pi} \times E_{g}$, where $E_{\pi}$ stands for the commodity space pertaining to private goods while $E_{g}$ is the commodity space of public goods.

The consumption set for the $i^{t / h}$ consumer is given by a subset $C^{i}$ of $E$ that describes those consumption bundles that can be realized. A consumption plan for the $i^{\text {th }}$ consumer is a bundle $x \in C^{i}$. A consumption plan specifies some amount of goods and labor, which the $i^{\text {th }}$ consumer is able to realize; thus the $i^{t / 2}$ consumption set $C^{i} \subset E$ is the collection of all these consumption plans.

Production refers to a process by which certain commodities (inputs) are transformed into different ones (outputs). A production plan for the $j^{\text {th }}$ firm, denoted by $y^{j} \in E$, specifies the amount of inputs that are required to obtain some outputs. A production set for the $j^{\text {th }}$ firm, denoted by $S^{j} \subset E$, is the collection of all the production plans that are possible for the $j^{\text {th }}$ firm according to the technological knowledge available to it.

\subsection{The Model}

The economy $\mathcal{E}$ under consideration consists of $n \in \mathbb{N}$ consumers with the corresponding consumption sets $C^{i}=C_{\pi}^{i} \times C_{g}^{i}$ as $i=1, \ldots, n$, where $C_{\pi}^{i} \subset E_{\pi}$ and $C_{g}^{i} \subset E_{g}$, and $m \in \mathbb{N}$ production sets $S^{j} \subset E=E_{\pi} \times E_{g}$ as $j=1, \ldots, m$. We suppose for simplicity that $C_{g}^{i}=C_{g}$ for all $i=1, \ldots, n$, i.e., everyone in the consumer sector chooses the same consumer bundles $x_{g}$ of public goods and different bundles of private goods. The results of this paper show, in particular, that each consumer faces different prices for public goods but the same price for all the private goods of the economy.

For each consumer $i \in\{1, \ldots, n\}$ we have the preference set

$$
P^{i}(x)=P_{\pi}^{i}\left(x_{\pi}\right) \times P_{g}^{i}\left(x_{g}\right) \subset C_{\pi}^{i} \times C_{g}^{i}
$$

defined as the collection of all elements in $C^{i}$.preferred to $x^{i}$ by this consumer at the consumption plan $x=\left(x^{i} \mid i=1, \ldots, n\right)$. It follows from the definition that $x^{i} \notin P^{i}(x)$ for all $i=1, \ldots, n$. In an economy with public goods it is natural to assume that at least one individual desires the public good, i.e., $P_{g}^{i}\left(x_{g}\right) \neq \emptyset$ for the corresponding index $i$. For convenience we put $\mathrm{cl} P^{i}(x)=\left\{x^{i}\right\}$ if $P^{i}(x)=\emptyset$. Observe that we do not use utility functions to describe preference sets and also do not impose any preordering and/or other conventional assumptions of welfare economics.

Let us finally consider a general net demand constraint set $W=W_{\pi} \times W_{g} \in E$ allowing us to describe market constraints of the economy and unify some conventional situations in economic modeling; cf. $[28,31]$ for welfare economies with only private goods. In the classical "markets clear" setting we have $W=(\omega, 0)$, where $\omega \in W_{\pi}$ is an aggregate initial 
endowment of scarce resources and $0 \in W_{g}$ means that there is no endowment of public goods. Another conventional situation corresponding to the so-called "implicit free disposal" of private commodities is modeled by $W_{g}=\{0\}$ and $W_{\pi}:=\omega-\left(E_{\pi}\right)_{+}$, where $\left(E_{\pi}\right)_{+}$stands for the closed positive cone of an ordered private commodity space $E_{\pi}$; see, e.g., $[9,20]$. In general the net demand set $W$ describes natural situations that may happen when the initial aggregate endowment is not exactly known due to, e.g., incomplete informa.tion. The latter particularly reflects uncertainties in economic modeling.

Next we define the notions of feasible allocations for the economy $\mathcal{E}$ under consideration.

Definition 2.1 (feasible allocations). We say that $(x, y) \in \prod_{i=1}^{n} C^{i} \times \prod_{i=1}^{n} S^{j}$ is a FEASIBLE ALLOCATION of $\mathcal{E}$ if the market constraint

$$
\sum_{i=1}^{n}\left(x_{\pi}^{i}, x_{g}\right)-\sum_{j=1}^{m} y^{j} \in W
$$

is satisfied with the given net demand constraint set $W$.

The main goal of this paper is to study the following Pareto-type notions of optimal allocations for $\mathcal{E}$ from the viewpoint of deriving necessary optimality conditions for them, which provide in fact extended versions of the second welfare theorem for economies with (private and) public goods.

Definition 2.2 (Pareto-type optimal allocations). Let $(\bar{x}, \bar{y})$ be a feasible allocation of the economy $\mathcal{E}$ with the local satiation property

$$
\bar{x}^{i} \in \operatorname{cl} P^{i}(\bar{x}) \text { for all } i=1, \ldots, n \text {. }
$$

Then: we say that:

(i) $(\bar{x}, \bar{y})$ is a WEAK PARETO OPTIMAL ALLOCATION of $\mathcal{E}$ if for any feasible allocation $(x, y)$ of $\mathcal{E}$ we have the relationships:

$$
x^{i} \notin P^{i}(\bar{x}) \text { for some index } i \in\{1, \ldots, n\} .
$$

(ii) $(\bar{x}, \bar{y})$ is a PARETo optimal ALLOCATION of $\mathcal{E}$ if for any feasible allocation $(x, y)$ of $\mathcal{E}$ we have the relationships:

either $x^{i} \notin \operatorname{cl} P^{i}(\bar{x})$ for some $i \in\{1, \ldots, n\}$ or $x^{i} \notin P^{i}(\bar{x})$ for all $i=1, \ldots, n$.

(ii) $(\bar{x}, \bar{y})$ is a STRONG PARETO OPTIMAL ALLOCATION of $\mathcal{E}$ if for any feasible allocation $(x, y)$ with $x \neq \bar{x}$ we have

$$
x^{i} \notin \mathrm{cl} P^{i}(\bar{x}) \text { for some } i \in\{1, \ldots, n\} .
$$

It is clear that (iii) $\Longrightarrow($ ii $) \Longrightarrow$ (i) but not vice versa. Note the notions of Pareto and weak Pareto optimal allocations are conventional in welfare economics. The notion of strong Pareto optimal allocations was introduced by Khan [19] and then was studied, e.g., in $[4,5,20,23,29,31]$, particularly for economies with only private goods. 
We can naturally define appropriate local versions of the above Pareto-type optimal allocations, but in this paper we confine ourselves with the study of the (global) versions from Definition 2.2. In the recent paper [4] the reader can find more discussions on the relationships between local and global Pareto-type notions and the corresponding versions of the extended second welfare theorem for economies with only private goods.

\subsection{Qualification Conditions}

We formulate and discuss here the mild qualification conditions introduced in [28] and then used in $[23,29,31]$ for studying Pareto and weak Pareto optimal allocations of welfare economies with only private goods.

Definition 2.3 (net demand qualification conditions). Let $(\bar{x}, \bar{y})$ be a feasible allocation of the economy $\mathcal{E}$, and let

$$
\bar{w}:=\sum_{i=1}^{n}\left(\bar{x}_{\pi}^{i}, \bar{x}_{g}\right)-\sum_{j=1}^{m} \bar{y}^{j}
$$

Given $\varepsilon>0$, consider the set

$$
\triangle_{\varepsilon}:=\sum_{i=1}^{n}\left[\operatorname{cl} P^{i}(\bar{x}) \cap\left(\bar{x}^{i}+\varepsilon \mathbb{B}\right)\right]-\sum_{j=1}^{m}\left[\operatorname{cl} S^{j} \cap\left(\bar{y}^{j}+\varepsilon \mathbb{B}\right)\right]-\operatorname{cl} W \cap(\bar{w}+\varepsilon \mathbb{B})
$$

and say that:

(i) The NET DEMAND WEAK QUALIFICATION (NDWQ) CONDITION holds at $(\bar{x}, \bar{y})$ if there are a number $\varepsilon>0$ and a sequence $\left\{e^{k}\right\} \subset E$ with $e^{k} \rightarrow 0$ as $k \rightarrow 0$ such that

$$
\triangle_{\varepsilon}+e^{k} \subset \sum_{i=1}^{n} P^{i}(\bar{x})-\sum_{j=1}^{m} S^{j}-W \text { for all large } k \in \mathbb{N} .
$$

(ii) The NET DEMAND QUALIFICATION (NDQ) CONDITION holds at $(\bar{x}, \bar{y})$ if there are a. number $\varepsilon>0$, a sequence $\left\{e^{k}\right\} \subset E$ with $e^{k} \rightarrow 0$ as $k \rightarrow 0$, and a consumer index $i_{0} \in\{1, \ldots, n\}$ such that

$$
\triangle_{\varepsilon}+e^{k} \subset P^{i_{1}}(\bar{x})+\sum_{i \neq i_{0}}^{n} \mathrm{cl} P^{i}(\bar{x})-\sum_{j=1}^{m} S^{j}-W \text { for all large } k \in \mathbb{N} .
$$

Since we obviously have the inclusion

$$
\sum_{i=1}^{n} P^{i}(\bar{x}) \subset P^{i_{0}}(\bar{x})+\sum_{i \neq i_{0}}^{n} \operatorname{cl} P^{i}(\bar{x})
$$

the NDWQ condition implies the NDQ one while, as it is easy to see, not vice versa. Note that for economies with only private goods and markets clear constraints-i.e., when $W=(\omega, 0) \in E_{\pi} \times E_{g}$ in $(2.1)$ - the NDQ condition was defined and applied in $[17,18]$ with discussing sufficient conditions for its validity that cover those from $[6,9]$ and other publications. Some extension of the NDQ condition has been recently introduced in [16] 
for private good economies; see also $[4,5]$ for further extensions and more discussions. In $[13,21,19,20,25,35,36]$ the reader can find qualification conditions for economies with public goods implying the NDQ condition from Definition 2.3.

Observe that the NDQ condition is designed to handle Pareto optimal allocations of welfare economies while the NDWQ one is more appropriate for the study of weak Pareto optimality; see below. We refer the reader to $[4,5,12]$ for some specifications of the NDWQ condition for weak Pareto optimal allocations and their relationships with Mas-Collel's uniform properness [24] and its modifications for economies with private goods.

To present next verifiable conditions ensuring the NDQ and NDWQ ones, we recall an important property of sets broadly used in the paper. A nonempty subset $\Omega \subset X$ of a normed space is epi-Lipschitzian around $\bar{x} \in \operatorname{cl} \Omega$ (in the sense of Rockafellar [32]) if there are neighborhoods $U$ of $\bar{x}$ and $O$ of $0 \in X$, a number $\gamma>0$, and a vector $c \in X$ such that

$$
\Omega \cap U+t O \subset \Omega+t c \text { for all } t \in(0, \gamma) \text {. }
$$

If the set $\Omega$ is closed around $\bar{x}$, property $(2.10)$ with $c \neq 0$ is equivalent to a local homeomorphic representation of $\Omega$ via the epigraph of a real-valued Lipschitz continuous functions; that as where the name comes from. It is worth mentioning that the closure of $\Omega$ is epiLipschitzian around $\bar{x} \in \operatorname{cl} \Omega$ if the set $\Omega$ enjoys this property around this point, but not vice versa. Furthermore, a convex set $\Omega$ is epi-Lipschitzian around each of its point if and only if int $\Omega \neq \emptyset$; see, e.g., [30, Proposition 1.25].

The following proposition gives sufficient conditions for the fulfillment of the NDWQ and NDQ properties of Definition 2.3.

Proposition 2.4 (sufficient conditions for the validity of net demand constraint qualifications). Let $(\bar{x}, \bar{y})$ be a feasible allocation of the economy $\mathcal{E}$ with public goods defined above. The following assertions holds:

(i) Assume that the sets $S^{j}, j=1, \ldots, m$, and, $W$ are closed around the points $\bar{y}^{j}$ and $\bar{w}$ from (2.1), respectively. Then the $N D Q$ condition is satisfied at $(\bar{x}, \bar{y})$ if there are $\varepsilon>0$, $i \in\{1, \ldots, n\}$, and a desirability sequence $\left\{e^{i k}\right\} \subset E$ with $e^{i k} \rightarrow 0$ as $k \rightarrow \infty$ such that

$$
\operatorname{cl} P^{i}(\bar{x}) \cap\left(\bar{x}^{i}+\varepsilon \mathbb{B}\right)+e^{i k} \subset P^{i}(\bar{x}) \text { for all large } k \in \mathbb{N} \text {. }
$$

Moreover, the NDWQ condition is satisfied at $(\bar{x}, \bar{y})$ if a desirability sequence $\left\{e^{i k}\right\}$ in (2.11) exists for each $i \in\{1, \ldots, n\}$ with some $\varepsilon>0$.

(ii) Assume that $\bar{x}^{i} \in \operatorname{cl} P^{i}(\bar{x})$ for all $i \in\{1, \ldots, n\}$. Then the $N D W Q$ condition is satisfied at $(\bar{x}, \bar{y})$ if the set

$$
\Delta:=\sum_{i=1}^{n} P^{i}(\bar{x})-\sum_{j=1}^{m} S^{j}-W
$$

is epi-Lipschitzian at $0 \in \mathrm{cl} \Delta$. It happens, in particular, when either one among the sets $P^{i}(\bar{x})$ for $i=1, \ldots, n, S^{j}$ for $j=1, \ldots, m$, and $W$ or some of their partial combinations in (2.12) is epi-Lipschitzian around the corresponding point. 
(iii) Assume that $n>1$. The $N D Q$ condition is satisfied at $(\bar{x}, \bar{y})$ if there is a consumer $i_{0} \in\{1, \ldots, n\}$ such that $P^{i_{0}}(\bar{x}) \neq \emptyset$ and the set

$$
\Sigma:=\sum_{i \neq i_{0}} \operatorname{cl} P^{i}(\bar{x})
$$

is epi-Lipschitzian at the point $\sum_{i \neq i_{0}} \bar{x}^{i}$. It happens, in particular, when either one among the sets $P^{i}(\bar{x})$ for $i \in\{1, \ldots, n\} \backslash\left\{i_{0}\right\}$ or some of their partial combinations in (2.13) is epi-Lipschitzian around the corresponding point.

Proof. Similar to that in [31, Proposition 8.4] for economies with private goods.

Note that condition (2.11) is a direct generalization of the desirability direction condition in [24], which is related to the classical "more is better" assumption for convex economies with only private goods and commodity spaces ordered by their closed positive cones having nonempty interiors. Furthermore, it is important to observe that we do not need to impose any assumption on the preference and/or production sets for the validity of both qualification conditions in Definition 2.3 if the net demand constraint set $W$ is epi-Lipschitzian around $\bar{w}$; this easily follows from Proposition 2.4(ii). The latter covers, in particular, the case of free-disposal Pareto optimum; see, e.g., $[9,20]$.

\section{Tools of Variational Analysis}

This section contains some constructions and preliminary results from variational analysis and generalized differentiation that are widely used in this paper to derive extended versions of the second welfare theorem in nonconvex economies with public goods. We mostly follow the book [30], where the reader can find all the proofs and more discussions.

\subsection{Generalized Normals}

We start with constructions of generalized normals to subsets of Banach spaces.

Definition 3.1 (generalized normals to sets). Let $\Omega$ be a nonempty subset of $E$.

(i) Given $x \in \Omega$ and $\varepsilon \geq 0$, the SET of $\varepsilon$-NORMALS to $\Omega$ at $x \in \Omega$ is defined by

$$
\widehat{N}_{\varepsilon}(x ; \Omega):=\left\{x^{*} \in E^{*} \mid \underset{u \stackrel{\operatorname{\Omega im}}{\rightarrow} x}{\operatorname{Limsup}_{x}} \frac{\left\langle x^{*}, u-x\right\rangle}{\|u-x\|} \leq \varepsilon\right\},
$$

where Limsup stands for the Painlevé-Kuratowski outer limit (1.1) and where the symbol $u \stackrel{\Omega}{\rightarrow} x$ means that $u \rightarrow x$ with $u \in \Omega$. When $\varepsilon=0$ in $(3.1)$, the cone $\widehat{N}(x ; \Omega):=\widehat{N}_{0}(x ; \Omega)$ is called the FRÉCHET NORMAL CONE to $\Omega$ at $x$.

(ii) Given, $\bar{x} \in \Omega$, the outer limit

$$
N(\bar{x} ; \Omega):=\operatorname{Limsup}_{\substack{x \rightarrow \frac{\Omega}{2} \bar{x} \\ \varepsilon \downarrow 0}} \widehat{N}_{\varepsilon}(x ; \Omega)
$$

is called the MORDUKHOVICH NORMAL CONE to $\Omega$ at $\bar{x}$. 
Note that construction (3.1) with $\varepsilon=0$ is also known in the literature as the prenormal or regular normal cone while (3.2) as basic, limiting, or $M$-normal cone. If $\Omega$ is locally closed around $\bar{x}$ and the space $E$ is $A$ splund (i.e., each of its separable subspace has a separable dual), then the normal cone (3.2) admits the simplified representation

$$
N(\bar{x} ; \Omega):=\operatorname{Limsup}_{x \rightarrow \frac{\Omega}{\rightarrow} \bar{x}} \widehat{N}(x ; \Omega) .
$$

The class of Asplund spaces is sufficiently large including, in particular, every reflexive Banach space and every space with a separable dual; see [30] for more details and references. If $E=\mathbb{R}^{n}$ and $\Omega$ is locally closed around $\bar{x}$, representation (3.3) is equivalent to the original definition in $[26]$ given by

$$
N(\bar{x} ; \Omega)=\operatorname{Limsup}_{x \rightarrow \bar{x}}[\operatorname{cone}(x-\Pi(x ; \Omega))]
$$

via the Euclidean projector $\Pi(x ; \Omega)$ of $x$ on $\Omega$, where the symbol 'cone' signifies the conic hull spanned on the set in question.

In the case of convex sets we have the following representations of generalized normals from Definition 3.1 showing, in particular, that both cones $\widehat{N}(\bar{x} ; \Omega)$ and $N(\bar{x} ; \Omega)$ under consideration extend the classical one in convex analysis.

Proposition 3.2 (normals to convex sets). Let $\Omega$ be convex. Then given arbitrary $\bar{x} \in \Omega$ and $\varepsilon \geq 0$, we have

$$
\widehat{N}_{\varepsilon}(\bar{x} ; \Omega)=\widehat{N}(\bar{x} ; \Omega)+\varepsilon \mathbb{B}^{*}=\left\{x^{*} \in E^{*} \mid\left\langle x^{*}, x-\bar{x}\right\rangle \leq \varepsilon\|x-\bar{x}\| \text { for all } x \in \Omega\right\},
$$

which implies the representations

$$
\widehat{N}(\bar{x} ; \Omega)=N(\bar{x} ; \Omega)=\left\{x^{*} \in E^{*} \mid\left\langle x^{*}, x-\bar{x}\right\rangle \leq 0 \text { for all } x \in \Omega\right\}
$$

Note that in the general nonconvex case the first equality of (3.5) is replaced by

$$
\widehat{N}_{\varepsilon}(\bar{x} ; \Omega) \supset \widehat{N}(\bar{x} ; \Omega)+\varepsilon \mathbb{B}^{*}, \quad \varepsilon>0 .
$$

In what follows we also employ the following useful formulas for representing generalized normals to products of arbitrary sets.

Proposition 3.3 (normal cones to products of sets). Let $\Omega_{i} \subset E_{i}, i=1,2$, be nonempty subsets of Banach spaces, and let $\bar{x}=\left(\bar{x}_{1}, \bar{x}_{2}\right) \in \Omega_{1} \times \Omega_{2}$. Then we have

$$
\begin{aligned}
& \widehat{N}\left(\bar{x} ; \Omega_{1} \times \Omega_{2}\right)=\widehat{N}\left(\bar{x}_{1} ; \Omega_{1}\right) \times \widehat{N}\left(\bar{x}_{2} ; \Omega_{2}\right), \\
& N\left(\bar{x} ; \Omega_{1} \times \Omega_{2}\right)=N\left(\bar{x}_{1} ; \Omega_{1}\right) \times N\left(\bar{x}_{2} ; \Omega_{2}\right)
\end{aligned}
$$

The next important results taken from [30, Theorem 1.30] provide smooth variational representations of Fréchet normals that play a crucial role in nonlinear price descriptions of decentralized equilibria in the extended second welfare theorems for economies with public good established in this paper. Note that assertion (i) of Theorem 3.4 below holds in arbitrary Banach space, while assertion (ii) gives an essentially stronger description of Fréchet normals under an additional geometric assumption on the space in question. 
Recall that a Banach space $E$ is Fréchet smooth if there is an equivalent norm on $E$ that is Fréchet differentiable at any nonzero point. In particular, every reflexive space is Fréchet smooth. Observe also that every Fréchet smooth space is Asplund, and hence we can use formula (3.3) for representing our basic normals.

Theorem 3.4 (smooth variational descriptions of Fréchet normals). Let $\Omega$ be $a$ nonempty subset of a Banach space $E$, and let $\bar{x} \in \Omega$. The following assertions hold:

(i) Given $x^{*} \in E^{*}$, assume that there is a function $s: U \rightarrow \mathbb{R}$ defined on a neighborhood of $\bar{x}$ and Fréchet differentiable at $\bar{x}$ such that $\nabla s(\bar{x})=x^{*}$ and $s(x)$ achieves a local maximum relative to $\Omega$ at $\bar{x}$. Then $x^{*} \in \widehat{N}(\bar{x} ; \Omega)$. Conversely, for every $x^{*} \in \widehat{N}(\bar{x} ; \Omega)$ there is a function $s: E \rightarrow \mathbb{R}$ such that $s(x) \leq s(\bar{x})=0$ whenever $x \in \Omega$ and that $s(\cdot)$ is Fréchet differentiable at $\bar{x}$ with $\nabla s(\bar{x})=x^{*}$.

(ii) Assume that $E$ is Fréchet smooth. Then for every $x^{*} \in \widehat{N}(\bar{x} ; \Omega)$ there is a concave Fréchet differentiable function $s: E \rightarrow \mathbb{R}$ that achieves its global maximum relative to $\Omega$ uniquely at $\bar{x}$ and such that $\nabla s(\bar{x})=x^{*}$.

One of the major features of the $M$-normal cone (3.2) is its nonconvexity, even in the case of rather simple nonconvex sets in finite dimensions, e.g., when $\Omega$ is either the graph of the function $|x|$ at $(0,0) \in \mathbb{R}^{2}$ or the epigraph of the function $-|x|$ at the origin. This does not allows us to employ conventional techniques of convex and 'convexified' analysis, mainly based on convex separation theorems and related results, to the study and applications of the $M$-normal cone and the associated constructions for functions and (single-valued and set-valued) mappings. Also the nonconvexity of (3.2) indicates that this normal cone is not dual/polar to any tangent cone, since polarity always implies convexity. Nevertheless, the $M$-normal cone and the corresponding subdifferential and coderivative constructions enjoy full calculus and other nice properties crucial in applications, mainly in the general framework of Asplund spaces; see $[30,31]$ and the references therein. These phenomena are based on advanced variational/extremal principles of modern variational analysis.

In the next subsection we present the basic extremal principle in Asplund space used in this paper for deriving extended versions of the second welfare theorem for nonconvex economies with infinite commodities and public goods.

\subsection{Extremal Principle}

First we present and briefly discuss the required definitions and then formulate the underlying results on the extremal principle. The reader can find full proofs, more discussions, and references in [30, Chapter 2] and the commentaries therein.

Definition 3.5 (local extremal points). Let $\Omega_{1}, \ldots, \Omega_{n}$ with $n \geq 2$ be nonempty subsets of a normed space $E$. We say that $\bar{x} \in \cap_{i=1}^{n} \Omega_{i}$ is a LOCAL EXTREMAL POINT of the set system $\left\{\Omega_{1}, \ldots, \Omega_{n}\right\}$ if there are sequences $\left\{a_{i}^{k}\right\} \subset E, i=1, \ldots, n$, and a neighborhood $U$ of $\bar{x}$ such that $a_{i}^{k} \rightarrow 0$ as $k \rightarrow \infty$ and that

$$
\bigcap_{i=1}^{n}\left(\Omega_{i}-a_{i}^{k}\right) \cap U=\emptyset \text { for all large } k \in \mathbb{N} .
$$


The system of sets having at least one local extremal point is called an EXTREMAL SYSTEM.

As shown in $[30,31]$ and their references, the concept of set extremality encompasses various notions of optimal solutions to problems of scalar and vector/multiobjective optimization. On the other hand, a number of nonvariational issues (e.g., calculus rules, stability, etc.) reduce to extremal systems of sets by using a variational approach. In this paper we show that the above notions of Pareto optimal allocations in economies with public goods can be reduced to extremal points of appropriate set systems under the imposed net demand qualification conditions for the cases Pareto and weak Pareto optimal allocations and with no such conditions for strong Pareto ones.

The next theorem contains two versions of the extremal principle used in what follows. The first version is approximate, which does not require any extra assumptions but expresses the result in terms of Fréchet normals to the sets $\Omega_{i}$ at points nearby the local extremal one. The second version provides an exact extremality condition formulated via the $M$-normal cone at the local extremal point in question under certain additional assumptions on the sets $\Omega_{i}$. These assumptions are automatic in finite dimensions while imposing a sufficient amount of "normal compactness" in infinite-dimensional spaces. In the sequel we use the following condition, perhaps the weakest one of this type needed for general systems of sets.

Definition 3.6 (sequential normal compactness). Let $\Omega \subset E_{1} \times E_{2}$ be a set in the product of two normed spaces. We say that:

(i) $\Omega$ is SEQUENTIALLY NORMALLY COMPACT (SNC) at $\bar{x} \in \Omega$ if for any sequences $\varepsilon_{k} \downarrow 0, x_{k} \rightarrow \bar{x}$ with $x_{k} \in \Omega$ satisfying

$$
\left(x_{k}^{* 1}, x_{k}^{* 2}\right) \in \widehat{N}_{\varepsilon_{k}}\left(x_{k} ; \Omega\right) \text { for all } k \in \mathbb{N}
$$

we have the implication

$$
\left(x_{k}^{* 1}, x_{k}^{* 2}\right) \stackrel{w^{*}}{\rightarrow}(0,0) \Longrightarrow\left\|\left(x_{k}^{* 1}, x_{k}^{* 2}\right)\right\| \rightarrow 0 \text { as } k \rightarrow \infty .
$$

(ii) $\Omega$ is PARTIALLY SNC (PSNC) at $\bar{x}$ with respect to the first component if for any sequences $\left(\varepsilon_{k}, x_{k}, x_{k}^{* 1}, x_{k}^{* 2}\right)$ from (i) we have the implication

$$
\left[x_{k}^{* 1} \stackrel{w^{*}}{\rightarrow} 0,\left\|x_{k}^{* 2}\right\| \rightarrow 0\right] \Longrightarrow\left\|x_{k}^{* 1}\right\| \rightarrow 0 \text { as } k \rightarrow \infty .
$$

(iii) $\Omega$ is STRONGLY PSNC at $\bar{x}$ with respect to the first component if for any sequences $\left(\varepsilon_{k}, x_{k}, x_{k}^{* 1}, x_{k}^{* 2}\right)$ from (i) we have the implication

$$
\left[\left(x_{k}^{* 1}, x_{k}^{* 2}\right) \stackrel{w^{*}}{\rightarrow}(0,0)\right] \Longrightarrow\left\|x_{k}^{* 1}\right\| \rightarrow 0 \text { as } k \rightarrow \infty .
$$

If $\Omega$ is a locally closed subset of an Asplund space, we can equivalently put $\varepsilon_{k} \equiv 0$ in all the relationships of Definition 3.6. Observe that, besides sets in finite-dimensional spaces, the SNC property holds for any subset of a Banach space that is compactly epiLipschitzian (CEL) around $\bar{x} \in \Omega$ in the sense of of Borwein and Strójwas [8], which means that a singleton $\{c\}$ in definition (2.10) of the epi-Lipschitzian property is replaced by some 
compact set $C \subset E$. More subtle conditions of the Lipschitzian type ensuring the PSNC and strong PSNC properties can be found in [30]

Now we are ready to formulate both approximate and exact versions of the extremal principle proved in [30, Theorem 2.20 and Theorem 2.22], respectively.

Theorem 3.7 (extremal principle in Asplund spaces). Let $\vec{x}$ be a local extre mal point of the set system $\left\{\Omega_{1}, \ldots, \Omega_{n}\right\}$ in an Asplund space $E$. Assume that all the sets $\Omega_{i}$ are locally closed around $\bar{x}$. Then the following assertions hold:

(i) For any $\varepsilon>0$ there are $x_{i} \in \Omega_{i} \cap(\bar{x}+\varepsilon \mathbb{B})$ and $x_{i}^{*} \in E^{*}$ satisfying the relationships

$$
\begin{gathered}
x_{i}^{*} \in \widehat{N}\left(x_{i} ; \Omega_{i}\right)+\varepsilon \mathbb{B}^{*} \text { for all } i=1, \ldots, n, \\
\qquad \sum_{i=1}^{n} x_{i}^{*}=0, \\
\sum_{i=1}^{n}\left\|x_{i}^{*}\right\|=1 .
\end{gathered}
$$

(ii) In addition to the assumptions above, suppose that all but one of the sets $\Omega_{i}$ as $i=1, \ldots, n$ are $S N C$ at $\vec{x}$. Then there are $M$-normals

$$
x_{i}^{*} \in N\left(\bar{x} ; \Omega_{i}\right) \text { for all } i=1, \ldots, n
$$

satisfying the relationships in (3.8) and (3.9).

It is easy to see from Proposition 3.2 that for the case of two convex sets $\Omega_{1}$ and $\Omega_{2}$ relationships (3.8)-(3.10) of the exact extremal principle reduce to conventional convex separation theorem, where the SNC requirement imposed on one of the sets is a far-going extension of the classical interiority condition, even in the case of convexity. Relationships (3.7)-(3.9) of the approximate extremal principle can be treated as a nonconvex counterpart of the celebrated Bishop-Phelps density theorem for convex sets with empty interiors; see [30, Section 2.1] for more discussions.

Combining these observations with the fact that the extremal principle gives necessary conditions for set extremality and thus can be considered an "extended Lagrange multipliers rule," our extremal principle approach to the second welfare theorem provides a unification of the classical foundation approach and the Arrow-Debreu separation/decomposition approach to welfare economics in general nonconvex and nonsmooth settings.

\section{Approximate Versions of the Extended Second Welfare Theorem for Economies with Public Goods}

In this section we derive necessary optimality conditions for Pareto and weak Pareto optimal allocations of welfare economies with public goods given in certain approximate forms under the underlying NDQ and NDWQ qualification conditions. The essence of such results, which provide extended versions of the second welfare theorem for convex and nonconvex 
economies, is that their formulations involve not only the optimal allocation in question, but also feasible allocations nearby. The results obtained in approximate forms hold with no extra assumptions on the initial data in infinite-dimensional commodity spaces.

We present two versions of the approximate second welfare theorem for economies with public goods mentioned in Section 1. Let us start with the first version, where (linear) marginal prices are formalized via. Fréchet normals. The results are given and proved in a parallel way for Pareto and weak Pareto optimal allocations.

Theorem 4.1 (extended second welfare theorem via approximate marginal prices). Let $(\bar{x}, \bar{y})$ be a Pareto (resp. weak Pareto) optimal allocation of the economy $\mathcal{E}$ with public goods. Assume that the commodity space $E$ is Asplund and that the $N D Q$ (resp. NDWQ) condition is satisfied at $(\bar{x}, \bar{y})$. Then given any $\varepsilon>0$, there are a commodity bundle $(x, y, w) \in \prod_{i=1}^{n} \mathrm{cl} P^{i}(\bar{x}) \times \prod_{j=1}^{m} \mathrm{cl} S^{j} \times \mathrm{cl} W$ as well as marginal prices $\left(p_{\pi}^{*}, p_{g}^{*}\right) \in E_{\pi}^{*} \times E_{g}^{*}$ and $p_{g}^{* i} \in E_{g}^{*}$ for $i=1, \ldots, n$ satisfying the relationships:

$$
\begin{gathered}
\sum_{i=1}^{n} p_{g}^{i *}=p_{g}^{*}, \\
-\left(p_{\pi}^{*}, p_{g}^{* i}\right) \in \widehat{N}\left(x^{i} ; \operatorname{cl} P^{i}(\bar{x})\right)+\varepsilon \mathbb{B}^{*}, \quad x^{i} \in \bar{x}^{i}+\varepsilon \mathbb{B}, \quad i=1, \ldots, n, \\
\left(p_{\pi}^{*}, p_{g}^{*}\right) \in \widehat{N}\left(y^{j} ; \operatorname{cl} S^{j}\right)+\varepsilon \mathbb{B}^{*}, \quad y^{j} \in \bar{y}^{j}+\varepsilon \mathbb{B}, \quad j=1, \ldots, m, \\
\left(p_{\pi}^{*}, p_{g}^{*}\right) \in \widehat{N}(w ; \operatorname{cl} W)+\varepsilon \mathbb{B}^{*}, \quad w \in \bar{w}+\varepsilon \mathbb{B}, \\
1-\varepsilon / 2 \leq \max \left\{\left\|\left(p_{\pi}^{*}, p_{g}^{* i}\right)\right\|_{i \in\{1, \ldots, n\}},\left\|p_{g}^{*}\right\|\right\} \leq 1+\varepsilon / 2
\end{gathered}
$$

Proof. Define the product space $X:=E^{n+m+1}$ equipped with the sum norm

$$
\left\|\left(x_{1}, \ldots, x_{n+m+1}\right)\right\|:=\left\|x_{1}\right\|+\ldots+\left\|x_{n+m+1}\right\| .
$$

In this case the corresponding dual norm on $X^{*}$ is given by

$$
\left\|\left(x_{1}^{*}, \ldots, x_{n+m+1}^{*}\right)\right\|=\max \left\{\left\|x_{1}^{*}\right\|, \ldots,\left\|x_{n+m+1}^{*}\right\|\right\} .
$$

Observe that the space $X$ is Asplund as a product of Asplund spaces.

To prove both results formulated in the theorem (for Pareto and weak Pareto optimal allocations), we use the approximate extremal principle in the Asplund space $X$ applied to the system of the two closed sets defined as follows:

$$
\begin{gathered}
\Omega_{1}:=\prod_{i=1}^{n}\left[\operatorname{cl} P^{i}(\bar{x}) \cap\left(\bar{x}^{i}+\varepsilon \mathbb{B}\right)\right] \times \prod_{j=1}^{m}\left[\operatorname{cl} S^{j} \cap\left(\bar{y}^{j}+\varepsilon \mathbb{B}\right)\right] \times[\operatorname{cl} W \cap(\bar{w}+\varepsilon \mathbb{B})], \\
\Omega_{2}:=\left\{(x, y, w) \in X \mid \sum_{i=1}^{n}\left(x_{\pi}^{i}, x_{g}\right)-\sum_{j=1}^{m} y^{j}-w=0\right\} .
\end{gathered}
$$

Let us check that $(\bar{x}, \bar{y}, \bar{w})$ is an extremal point of the set system $\left\{\Omega_{1}, \Omega_{2}\right\}$ defined in (4.7) and (4.8). Indeed, we have by (2.1) and (2.2) that $(\bar{x}, \bar{y}, \bar{w}) \in \Omega_{1} \cap \Omega_{2}$. It remains to show that there is a sequence $\left\{a^{k}\right\} \subset X$ with $a^{k} \rightarrow 0$ as $k \rightarrow \infty$ such that

$$
\left(\Omega_{1}-a^{k}\right) \cap \Omega_{2}=\emptyset
$$


in the case of the Pareto (resp. weak Pareto) optimal allocation $(\bar{x}, \bar{y})$ of $\mathcal{E}$ under the fulfillment of the NDQ (resp. NDWQ) qualification requirements from Definition 2.3.

Let $\varepsilon>0$ and $\left\{e^{k}\right\} \subset E$ be such that the corresponding condition (2.8) and (2.9) is satisfied. Form a sequence $\left\{a^{k}\right\} \subset X$ by

$$
a^{k}:=\left(0, \ldots, 0, e^{k}\right) \text { for all } k \in \mathbb{N}
$$

and get by construction that $a^{k} \rightarrow 0$ as $k \rightarrow \infty$. Arguing by contradiction, suppose that relationship (4.9) does not hold along a subsequence of $k \rightarrow \infty$. Then we find triples $\left(x^{k}, y^{k}, w^{k}\right) \in \Omega_{1}$ such that $\left(x^{k}, y^{k}, w^{k}\right)-a^{k} \in \Omega_{2}$ for the corresponding numbers $k \in \mathbb{N}$. It follows from the constructions in (4.7), (4.8), and (4.10) that

$$
\begin{aligned}
& x^{k i} \in \operatorname{cl} P^{i}(\bar{x}) \cap\left(\bar{x}^{i}+\varepsilon \mathbb{B}\right), \quad i=1, \ldots, n, \\
& y^{k j} \in \operatorname{cl} S^{j} \cap\left(\bar{y}^{j}+\varepsilon \mathbb{B}\right), \quad j=1, \ldots, m, \\
& w^{k} \in \operatorname{cl} W \cap(\bar{w}+\varepsilon \mathbb{B}), \quad \text { and } \\
& \sum_{i=1}^{n}\left(x_{\pi}^{k i}, x_{g}^{k}\right)-\sum_{j=1}^{m} y^{k j}-w^{k}+e^{k}=0
\end{aligned}
$$

for $k \in \mathbb{N}$ sufficiently large. Comparing (4.11) with (2.8), we have

$$
0 \in \sum_{i=1}^{n} P^{i}(\bar{x})-\sum_{j=1}^{m} S^{j}-W
$$

which contradicts the weak Pareto optimality of the allocation $(\bar{x}, \bar{y})$. The comparison of (4.11) with (2.9) gives us the inclusion

$$
0 \in P^{i_{0}}(\bar{x})+\sum_{i \neq i_{0}}^{n} \operatorname{cl} P^{i}(\bar{x})-\sum_{j=1}^{m} S^{j}-W,
$$

which contradicts the Pareto optimality of $(\bar{x}, \bar{y})$. Thus $(\bar{x}, \bar{y}, \bar{w})$ is an extremal point of the set system $\left\{\Omega_{1}, \Omega_{2}\right\}$ in both cases under consideration.

Applying the extremal principle from assertion (i) of Theorem 3.7 to the system $\left\{\Omega_{1}, \Omega_{2}\right\}$ at $(\bar{x}, \bar{y}, \bar{w})$, for any $\varepsilon>0$ we find elements $u=\left(x^{1}, \ldots, x^{n}, y^{1}, \ldots, y^{m}, w\right) \in \Omega_{1}, v \in \Omega_{2}$, $x^{*} \in X^{*}$ with $\left\|x^{*}\right\|=1$, and

$$
u^{*} \in \widehat{N}\left(u ; \Omega_{1}\right), \quad v^{*} \in \widehat{N}\left(v ; \Omega_{2}\right)
$$

satisfying the relationships

$$
\begin{aligned}
& x^{*}-u^{*} \in(\varepsilon / 2) \mathbb{B}^{*}, \quad-x^{*}-v^{*} \in(\varepsilon / 2) \mathbb{B}^{*}, \\
& x^{i} \in \bar{x}^{i}+(\varepsilon / 2) \mathbb{B} \text { for } i=1, \ldots, n, \\
& y^{j} \in \bar{y}^{j}+(\varepsilon / 2) \mathbb{B} \text { for } j=1, \ldots, m, \\
& w \in \bar{w}+(\varepsilon / 2) \mathbb{B}, \text { and }
\end{aligned}
$$




$$
1-\varepsilon / 2 \leq\left\|v^{*}\right\| \leq 1+\varepsilon / 2, \quad\left\|v^{*}+u^{*}\right\| \leq \varepsilon .
$$

Since the set $\Omega_{2}$ in (4.8) is a linear subspace of $X$, we get by (3.6) and the choice of $\left(v, v^{*}\right)$ in (4.13) the equality

$$
\left\langle v^{*}, \theta\right\rangle=\left\langle v^{*}, v\right\rangle \text { for all } \theta \in \Omega_{2} .
$$

Let us now show that there are prices $p_{\pi}^{*} \in E_{\pi}^{*}$ and $p_{g}^{* i} \in E_{g}^{*}$ for $i=1, \ldots, n$ such that the vector $v^{*}$ in (4.15) admits the representation

$$
v^{*}=\left(\left(p_{\pi}^{*}, p_{g}^{* 1}\right), \ldots,\left(p_{\pi}^{*}, p_{g}^{* n}\right),-\left(p_{\pi}^{*}, p_{g}^{*}\right), \ldots,-\left(p_{\pi}^{*}, p_{g}^{*}\right),-\left(p_{\pi}^{*}, p_{g}^{*}\right)\right),
$$

where $p_{g}^{*}$ is given by (4.1). Indeed, writing $v^{*} \in\left(E^{n+m+1}\right)^{*}$ as

$$
v^{*}=\left(\left(x_{\pi}^{* 1}, x_{g}^{* 1}\right), \ldots,\left(x_{\pi}^{* n}, x_{g}^{* n}\right),\left(y_{\pi}^{* 1}, y_{g}^{* 1}\right), \ldots,\left(y_{\pi}^{* m}, y_{g}^{* m}\right),\left(w_{\pi}^{*}, w_{g}^{*}\right)\right),
$$

it is not hard to observe from (4.15) and the structure of the set $\Omega_{2}$ in (4.8) that

$$
\begin{gathered}
\sum_{i=1}^{n}\left\langle x_{g}^{* i}, z\right\rangle+\left\langle w_{g}^{*}, z\right\rangle=0 \\
\left\langle x_{\pi}^{* i}, z\right\rangle+\left\langle y_{\pi}^{* j}, z\right\rangle=0, \quad\left\langle x_{\pi}^{* i}, z\right\rangle+\left\langle w_{\pi}^{*}, z\right\rangle=0 \text { for all } i=1, \ldots, n \text { and } j=1, \ldots, m
\end{gathered}
$$

whenever vector $z \in E_{g}$ is chosen. The latter clearly gives that

$$
w_{g}^{*}=-\sum_{i=1}^{n} x_{g}^{* i} \text { and } x_{\pi}^{* i}=-y_{\pi}^{* j}=-w_{\pi}^{*} \text { for all such } i, j .
$$

In the same way we get that

$$
\sum_{i=1}^{n}\left\langle x_{g}^{* i}, z\right\rangle+\left\langle y_{g}^{* j}, z\right\rangle=0 \text { for all } j \in\{1, \ldots, m\} \text { and } z \in E_{g}
$$

which implies the relationships

$$
y_{g}^{* j}=-\sum_{i=1}^{n} x_{g}^{* i} \text { as } j=1, \ldots, m .
$$

Taking into account that $x_{\pi}^{* i}, y_{\pi}^{* j}$, and $y_{g}^{* j}$ are in fact independent of $i$ and $j$ by (4.18) and (4.19), respectively, and denoting

$$
p_{\pi}^{*}:=x_{\pi}^{* i}, \quad p_{g}^{*}:=y_{g}^{* j}, \quad \text { and } p_{g}^{* i}:=x_{g}^{* i} \text { for } i=1, \ldots, n,
$$

we get from (4.17), (4.18), and (4.19) the claimed representation (4.16) of the normal vector $v^{*}$ from (4.18), where the public goods price $p_{g}^{*}$ satisfies (4.1).

It follows from the first relationship in (4.14), representation (4.16), and the form of the dual norm (4.6) that the nontriviality estimates in (4.5) hold. From the second relationship in (4.14) we further get that

$$
-v^{*} \subset u^{*}+(\varepsilon / 2) \mathbb{B}^{*} \subset \widehat{N}\left(u ; \Omega_{1}\right)+\varepsilon \mathbb{B}^{*},
$$


which implies by (4.16) that

$$
-v^{*}=\left(-\left(p_{\pi}^{*}, p_{g}^{* 1}\right), \ldots,-\left(p_{\pi}^{*}, p_{g}^{* n}\right),\left(p_{\pi}^{*}, p_{g}^{*}\right), \ldots,\left(p_{\pi}^{*}, p_{g}^{*}\right),\left(p_{\pi}^{*}, p_{g}^{*}\right)\right) \in \hat{N}\left(u ; \Omega_{1}\right)+\varepsilon \mathbb{B}^{*}
$$

Employing the first product formula in Proposition 3.3 to the set $\Omega_{1}$ in (4.7) and observing that all the above triples $\left(x^{i}, y^{j}, w\right)$ belong to the corresponding $\varepsilon$-neighborhoods of the optimal one $(\bar{x}, \bar{y}, \bar{w})$, we conclude from the latter inclusion that all the relationships in (4.2)-(4.4) are satisfied, which completes the proof of the theorem.

Observe that the equality relationship (4.1) between the marginal prices in the public goods sector confirms and extends the fundamental conclusion of welfare economics with public goods that goes back to Samuelson [35]: the marginal rates of transformation for public goods equal to the sum of the individual marginal rates of substitution at Pareto and weak Pareto optimal allocations.

Let us present a specification of Theorem 4.1 in the conventional case with no initial endowment of public goods, i.e., when $W=W_{\pi} \times\{0\}$.

Corollary 4.2 (approximate marginal prices for economies with no initial endowment of public goods). Let $(\bar{x}, \bar{y})$ be a Pareto (resp. weak Pareto) optimal allocation of the economy $\mathcal{E}$ with $W=W_{\pi} \times\{0\}, W_{\pi} \subset E_{\pi}$, under the corresponding assumptions of Theorem 4.1. Then for any $\varepsilon>0$ there are $(x, y, w) \in \prod_{i=1}^{n} \mathrm{cl} P^{i}(\bar{x}) \times \prod_{j=1}^{m} \mathrm{cl} S^{j} \times \mathrm{cl} W$ as well as marginal prices $\left(p_{\pi}^{*}, p_{g}^{*}\right) \in E_{\pi}^{*} \times E_{g}^{*}$ and $p_{g}^{* i} \in E_{g}^{*}$ for $i=1, \ldots, n$ satisfying relationships (4.1)-(4.3) and (4.5) with the replacement of (4.4) by

$$
p_{\pi}^{*} \in \widehat{N}\left(w_{\pi} ; \operatorname{cl} W_{\pi}\right)+\varepsilon \mathbb{B}^{*}, \quad w_{\pi} \in \bar{w}_{\pi}+\varepsilon \mathbb{B} .
$$

Proof. Follows directly from Theorem 4.1 and Proposition 3.3.

The next consequence of Theorem 4.1 gives a specification of the results in the case of convexity assumptions imposed on preference and production sets. In this case the marginal price relationships reduce, respectively, to global minimization (maximization) of the perturbed consumer expenditures (firm profits) over the corresponding preference (production) sets. This provides an approximate decentralized price equilibrium in convex models with no standard interiority assumptions.

Corollary 4.3 (approximate decentralized equilibrium in convex economies with public goods). In the framework of Theorem 4.1, assume that the preference sets $\mathrm{cl} P^{i}(\bar{x})$, $i=1, \ldots, n$, and the production sets $S^{j}, j=1, \ldots, m$, are convex. Then for any $\varepsilon>0$ there exist $(x, y, w) \in \prod_{i=1}^{n} \mathrm{cl} P^{i}(\bar{x}) \times \prod_{j}^{m} \mathrm{cl} S^{j} \times \mathrm{cl} W$ and prices $\left(p_{\pi}^{*}, p_{g}^{*}\right) \in E_{\pi}^{*} \times E_{g}^{*}$ and $p_{g}^{* i} \in E_{g}^{*}$ for $i=1, \ldots, n$ satisfying relationships (4.1), (4.4), (4.5), and the following ones:

$$
\begin{gathered}
\left\langle\left(p_{\pi}^{*}, p_{g}^{* i}\right),(u, v)-\left(x_{\pi}^{i}, x_{g}\right)\right\rangle \geq-\varepsilon\left\|(u, v)-\left(x_{\pi}^{i}, x_{g}\right)\right\| \text { for all }(u, v) \in \operatorname{cl} P^{i}(\bar{x}), i=1, \ldots, n, \\
\left\langle\left(p_{\pi}^{*}, p_{g}^{*}\right),(u, v)-\left(y_{\pi}^{j}, y_{g}^{j}\right)\right\rangle \leq \varepsilon\left\|(u, v)-\left(y_{\pi}^{j}, y_{g}^{j}\right)\right\| \text { for all }(u, v) \in \operatorname{cl} S^{j}, j=1, \ldots, m .
\end{gathered}
$$


Proof. Follows from conditions (4.2) and (4.3) of Theorem 4.1 and the representation of $\varepsilon$-normals to convex sets given in Proposition 3.2 .

The next theorem, developing the corresponding results of $[29,31]$ to economies with public goods, establishes a decentralized price equilibrium of the convex type as in Corollary 4.3 but for general nonconvex models. The "price to pay" for this is the usage of nonlinear prices in nonconvex models instead of conventional linear prices as in Theorem 4.1 and Corollary 4.3. Note that the essence of nonlinear prices used here as well as in $[29,31]$ is different from that of [2] and related publications dealing with convex economies.

Theorem 4.4 (decentralized approximate equilibrium in nonconvex models with public goods via nonlinear prices). Given any $\varepsilon>0$, the following assertions hold:

(i) Let all the assumptions of Theorem 4.1 be fulfilled for a Pareto (resp. weak Pareto) optimal allocation $(\bar{x}, \bar{y})$ of the economy $\mathcal{E}$. Then there exist a commodity bundle $(x, y, w) \in$ $\prod_{i=1}^{n} \operatorname{cl} P^{i}(\bar{x}) \times \prod_{j}^{m} \operatorname{cl} S^{j} \times \operatorname{cl} W$, marginal prices $\left(p_{\pi}^{*}, p_{g}^{*}\right) \in E_{\pi}^{*} \times E_{g}^{*}$ and $p_{g}^{* i} \in E_{g}^{*}$ as $i=1, \ldots, n$ satisfying relationships (4.1), (4.4), and (4.5) as well as real-valued functions $f^{i}$ as $i=1, \ldots, n$ and $h^{j}$ as $j=1, \ldots, m+1$ on $E$ that are Fréchet differentiable at $x^{i}, y^{j}$, and $w$, respectively, with

$$
\left\{\begin{array}{l}
\left\|\nabla f^{i}\left(x^{i}\right)-\left(p_{\pi}^{*}, p_{g}^{* i}\right)\right\| \leq \varepsilon, \quad i=1, \ldots, n \\
\left\|\nabla h^{j}\left(y^{j}\right)-\left(p_{\pi}^{*}, p_{g}^{*}\right)\right\| \leq \varepsilon, \quad j=1, \ldots, m \\
\left\|\nabla h^{m+1}(w)-\left(p_{\pi}^{*}, p_{g}^{*}\right)\right\| \leq \varepsilon
\end{array}\right.
$$

and such that each $f^{i}, i=1, \ldots, n$, achieves its global minimum over $\mathrm{cl} P^{i}(\bar{x})$ at $x^{i}$, each $h^{j}, j=1, \ldots, m$, achieves its global maximum over $\mathrm{cl} S^{j}$ at $y^{j}$, and $h^{m+1}$ achieves its global maximum over $\mathrm{cl} W$ at $w$.

(ii) If in addition to the assumptions in (i) the commodity space $E$ is Fréchet smooth, then the nonlinear prices $f^{i}$ and $h^{j}$ can be chosen to be Fréchet differentiable on $E$ and such that each $f^{i}, i=1, \ldots, n$, is convex and achieves its global minimum over $\operatorname{cl} P^{i}(\bar{x})$ uniquely at $x^{i}$ while each $h^{j}, j=1, \ldots, m+1$, is concave and achieves its global maximum over $\mathrm{cl} S^{j}$ for $j=1, \ldots, m$ and over $\mathrm{cl} W$ for $j=m+1$ uniquely at $y^{j}$ and $w$, respectively.

Proof. Take the marginal prices $\left(p_{\pi}^{*}, p_{g}^{*}, p_{g}^{i *}\right)$ satisfying all the conclusions of Theorem 4.1 and then, by $(4.2)$ and $(4.3)$, find $\left(\widetilde{p}_{\pi}^{*}, \widetilde{p}_{g}^{*}, \widetilde{p}_{g}^{i *}\right)$ such that

$$
\left\{\begin{array}{l}
-\left(\widetilde{p}_{\pi}^{*}, \widetilde{p}_{g}^{* i}\right) \in \hat{N}\left(x^{i} ; \operatorname{cl} P^{i}(\bar{x})\right), \quad\left\|\left(\widetilde{p}_{\pi}^{*}, \widetilde{p}_{g}^{* i}\right)-\left(p_{\pi}^{*}, p_{g}^{* i}\right)\right\| \leq \varepsilon \text { for } i=1, \ldots, n, \\
\left(\widetilde{p}_{\pi}^{*}, \widetilde{p}_{g}^{*}\right) \in \hat{N}\left(y^{j} ; \operatorname{cl} S^{j}\right), \quad\left\|\left(\widetilde{p}_{\pi}^{*}, \widetilde{p}_{g}^{*}\right)-\left(p_{\pi}^{*}, p_{g}^{*}\right)\right\| \leq \varepsilon \text { for } j=1, \ldots, m, \\
\left(\widetilde{p}_{\pi}^{*}, \widetilde{p}_{g}^{*}\right) \in \hat{N}(w ; \operatorname{cl} W), \quad\left\|\left(\widetilde{p}_{\pi}^{*}, \widetilde{p}_{g}^{*}\right)-\left(p_{\pi}^{*}, p_{g}^{*}\right)\right\| \leq \varepsilon .
\end{array}\right.
$$

Applying now the smooth variational descriptions of Fréchet normals in (4.21) from assertions (i) and (ii) Theorem 3.4, we complete the proof of this theorem. 


\section{Exact Versions of the Extended Second Welfare Theorem for Economies with Public Goods}

In this section we establish necessary optimality conditions for Pareto and weak Pareto optimal allocations of the nonconvex economy $\mathcal{E}$ with public goods in the exact/pointwise form of the extended second welfare theorem under additional SNC assumptions imposed on the sets involved in the description of the economy $\mathcal{E}$. Note that SNC property and its partial modifications seem to be the weakest among compactness-like requirements needed for exact forms of the second welfare theorem. As mentioned in Section 3 and fully discussed in [30, Subsection 1.1.4], the basic SNC property is generally weaker that the CEL assumption imposed in the corresponding extensions $[12,17,18]$ for economies with only private goods. In this way we get improvements of the second welfare theorem even in the classical settings of convex economies with both private and public goods. We also present a decentralized version of the exact second welfare theorem for nonconvex economies via nonlinear prices.

We begin with the basic version of the second welfare theorem with marginal prices formalized via the $M$-normal cone (3.2) at Pareto and weak Pareto optimal allocations.

Theorem 5.1 (exact form of the extended second welfare theorem via marginal prices). Let $(\bar{x}, \bar{y})$ be a Pareto (resp. weak Pareto) optimal allocation of the economy $\mathcal{E}$ with public goods satisfying the corresponding assumptions of Theorem 4.1. Assume in addition that the preference sets $\mathrm{cl} P^{i}(\bar{x})$ are $S N C$ at $\bar{x}^{i}$ for all $i=1, \ldots, n$. Then there exist marginal prices $\left(p_{\pi}^{*}, p_{g}^{*}\right) \in E_{\pi}^{*} \times E_{g}^{*}$ and $p_{g}^{* i} \in E_{g}^{*}$ as $i=1, \ldots, n$ with

$$
\left(p_{\pi}^{*}, p_{g}^{* i}\right) \neq 0 \text { for at least one } i \in\{1, \ldots, n\}
$$

satisfying the normal cone inclusions

$$
\begin{gathered}
-\left(p_{\pi}^{*}, p_{g}^{* i}\right) \in N\left(\bar{x}^{i} ; \operatorname{cl} P^{i}(\bar{x})\right), \quad i=1, \ldots, n, \\
\left(p_{\pi}^{*}, p_{g}^{*}\right) \in N\left(\bar{y}^{j} ; \operatorname{cl} S^{j}\right), \quad j=1, \ldots, m, \\
\left(p_{\pi}^{*}, p_{g}^{*}\right) \in N(\bar{w} ; \operatorname{cl} W),
\end{gathered}
$$

and the underlying equality (4.1) for the prices associated with public goods.

Proof. We know from the proof of Theorem 4.1 that $(\bar{x}, \bar{y}, \bar{w})$ is an extremal point of the systems of sets $\left\{\Omega_{1}, \Omega_{2}\right\}$ defined in (4.7) and (4.8) under the NDQ (resp. NDWQ) condition in the case of Pareto (resp. weak Pareto) optimal allocations of the economy $\mathcal{E}$. To get a pointwise version of extended second welfare theorem, we can apply the exact extremal principle from assertion (ii) of Theorem 3.7. In this way we obtain, similarly to the proof of Theorem 4.1, the conclusions of Theorem 5.1 under consideration in the case of finitedimensional commodity spaces. However, in infinite dimensions this approach requires imposing the SNC assumption on all of the sets

$$
\operatorname{cl} P^{i}(\bar{x}), i=1, \ldots, n ; \quad \operatorname{cl} S^{j}, j=1, \ldots, m ; \quad \operatorname{cl} W .
$$


In what follows we do not apply the exact extremal principle directly but pass to the limit from the results of Theorem 4.1 based on the approximate version of the extremal principle. This allows us to arrive at all the conclusions (4.1), (5.1), (5.2)-(5.4) of Theorem 5.1 under less restrictive SNC assumptions made; see also Remark 5.2 below.

To proceed, take any sequence of $\varepsilon_{k} \downarrow 0$ as $k \rightarrow \infty$ and find by Theorem 4.1 triples

$$
\left(x^{k}, y^{k}, w^{k}\right) \in \prod_{i=1}^{n} \operatorname{cl} P^{i}(\bar{x}) \times \prod_{j=1}^{m} \operatorname{cl} S^{j} \times \operatorname{cl} W \text { with }\left(x^{k}, y^{k}, w^{k}\right) \rightarrow(\bar{x}, \bar{y}, \bar{w}) \text { as } k \rightarrow \infty
$$

and prices $\left(p_{\pi}^{* k}, p_{g}^{* k}, p_{g}^{* i k}\right)$ satisfying all the conclusions of Theorem 4.1 for $\varepsilon=\varepsilon_{k}$.

It follows from (4.5) that the price sequences $\left\{\left(p_{\pi}^{k}, p_{g}^{* i k}\right)\right\}$ are bounded for all $i=1, \ldots, n$. Taking into account that the commodity space $E$ is Asplund and hence any bounded subset of $E^{*}$ is sequentially compact in the weak* topology of $E^{*}$, we find $\left(p_{\pi}^{*}, p^{* i}\right)$ such that

$$
\left(p_{\pi}^{* k}, p_{g}^{* i k}\right) \stackrel{w^{*}}{\rightarrow}\left(p_{\pi}^{*}, p_{g}^{* i}\right) \text { as } k \rightarrow \infty, \quad i=1, \ldots, n
$$

along some subsequences, without relabeling. Setting

$$
p_{g}^{*}:=\sum_{i=1}^{n} p_{g}^{* i}
$$

and passing to the weak* limit in the equality

$$
p_{g}^{* k}:=\sum_{i=1}^{n} p_{g}^{* i k}, \quad k \in \mathbb{N}
$$

we get that $p_{g}^{* k} \rightarrow p_{g}^{*}$ as $k \rightarrow \infty$. Passing further to the weak* in the relationships (4.2)(4.4) with $\varepsilon=\varepsilon_{k}$ as $k \rightarrow \infty$ and using definition (3.2) of the $M$-nomal cone allow us to conclude that the limiting prices $\left(p_{\pi}^{*}, p_{g}^{*}, p_{g}^{* i}\right)$ satisfy the relationships in (5.2)-(5.4).

It remains to justify the nontriviality condition (5.1) under the SNC requirements imposed in the theorem. To proceed, assume the contrary, i.e.,

$$
p_{\pi}^{*}=0 \text { and } p_{g}^{* i}=0 \text { for all } i=1, \ldots, n .
$$

Then we have by (5.6) that

$$
\left(p_{\pi}^{* k}, p_{g}^{* i k}\right) \stackrel{w^{*}}{\rightarrow}(0,0) \text { as } k \rightarrow \infty, \quad i=1, \ldots, n .
$$

This implies by the SNC assumptions imposed in the theorem that

$$
\left\|\left(p_{\pi}^{* k}, p_{g}^{* i k}\right)\right\| \rightarrow(0,0) \text { as } k \rightarrow \infty, \quad i=1, \ldots, n .
$$

It follows from (5.7) and (4.1) that $\left\|p_{g}^{* k}\right\| \rightarrow 0$ as $k \rightarrow \infty$. The latter combined with (5.7) contradicts (4.5) as $\varepsilon=\varepsilon_{k}$ for large $k \in \mathbb{N}$ and thus completes the proof.

Remark 5.2 (SNC assumptions). It follows from the proof of Theorem 5.1 and Definition 3.6(ii,iii) that the SNC requirements on the preference sets $\operatorname{cl} P_{i}(\bar{x})$ in the theorem can 
be relaxed to keep the nontriviality condition (5.1) with taking into account the product structure of the commodity space $E=E_{\pi} \times E_{g}$. Indeed, it is sufficient to assume that:

- either one of the sets in (5.3) and (5.4) is strongly PSNC with respect of the first component while all the sets in (5.2) are PSNC with respect to the second component at the corresponding points;

- or all the sets in (5.2) are strongly PSNC with respect to the second component while one of the sets in (5.3) and (5.4) is PSNC with respect to the first component at the corresponding points.

Observe that in the case of economies with only private goods both requirements above reduce to imposing the SNC property on one of the set in (5.5) at the corresponding point, which is the content of [31, Theorem 8.8].

Next we present two useful specifications of Theorem 5.1 for economies with public goods and additional structural requirements on their initial data. The first one concerns economies with a special structure of the net demand constraint set, which includes the case of implicit free disposal of commodities.

Corollary 5.3 (excess demand condition). Suppose that in the setting of Theorem 5.1 the net demand constraint set $W \subset E_{\pi} \times E_{g}$ admits the representation

$$
W=\left(\omega_{\pi}, \omega_{g}\right)+\operatorname{cl} \Gamma
$$

where $\left(\omega_{\pi}, \omega_{g}\right) \in \mathrm{cl} W$ and $\Gamma$ is a nonempty convex subcone of $E_{\pi} \times \dot{E}_{g}$. There there are marginal prices $\left(p_{\pi}^{*}, p_{g}^{*}\right) \in E_{\pi}^{*} \times E_{g}^{*}$ and $p_{g}^{* i} \in E_{g}^{*}$ as $i=1, \ldots, n$ satisfying all the relationships in (4.1), (5.1)-(5.3) and such that

$$
\left\langle\left(p_{\pi}^{*} ; p_{g}^{*}\right), \sum_{i=1}^{n}\left(\bar{x}_{\pi}^{i}, \bar{x}_{g}^{i}\right)-\sum_{j=1}^{m} \bar{y}^{j}-\left(\omega_{\pi}, \omega_{g}\right)\right\rangle=0 .
$$

Proof. By Theorem 5.1 it remains to show that inclusion (5.4) for the special conic structure of $W$ in (5.8) implies the zero value of excess demand condition (5.9) at marginal prices, which is an economic manifestation of the complementary slackness condition in optimization. To proceed, observe from relationships (5.4), (5.8) and the normal cone representation (3.6) for convex sets that

$$
\left\langle\left(p_{\pi}^{*}, p_{g}^{*}\right), \bar{w}-\left(\omega_{\pi}, \omega_{g}\right)\right\rangle \geq\left\langle\left(p_{\pi}^{*}, p_{g}^{*}\right), w-\left(\omega_{\pi}, \omega_{g}\right)\right\rangle \text { for all } w \in \operatorname{cl} W .
$$

Hence $\left\langle\left(p_{\pi}^{*}, p_{g}^{*}\right),\left(\bar{w}-\left(\omega_{\pi}, \omega_{g}\right)\right\rangle \geq 0\right.$. On the other hand, we have

$$
2\left(\bar{w}-\left(\omega_{\pi}, \omega_{g}\right)\right) \in W-\left(\omega_{\pi}, \omega_{g}\right)=\Gamma
$$

due to the conic structure of $\Gamma$, which implies by $(5.10)$ that $\left\langle\left(p_{\pi}^{*}, p_{g}^{*}\right), \bar{w}-\left(\omega_{\pi}, \omega_{g}\right)\right\rangle \leq 0$. This yields (5.9) and completes the proof of the corollary.

In the case of economies with convex preference and production sets considered in the next corollary of Theorem 5.1, relationships (5.2) and 5.3-unified with those in (4.1) and (5.2) for prices corresponding to public goods-reduce to the classical consumer expenditure 
minimization and firm profit maximization conditions of the second fundamental theorem of welfare economics. We are able, however, to significantly improve the classical interiority condition required for the validity of the second welfare theorem with infinite commodities in convex settings. As known from [30, Theorem 1.17], the SNC property imposed in our Theorem 5.1 is equivalent, in the case of convex sets with nonempty relative interiors, to their finite codimension. Furthermore, convex sets in Asplund spaces may be SNC even having empty relative interiors; see [30, Example 3.6]. The PSNC extensions discussed above in Remark 5.2 signify further far-going departures from the interiority condition.

Corollary 5.4 (second welfare theorem in convex settings with no interiority requirements). In addition to the assumptions of Theorem 5:1, suppose that the sets $\operatorname{cl} P^{i}(\bar{x})$ as $i=1, \ldots, n$ and $\mathrm{cl} S^{j}$ as $j=1, \ldots, m$ are convex. Then there are prices $\left(p_{\pi}^{*}, p_{g}^{*}\right) \in$ $E_{\pi}^{*} \times E_{g}^{*}$ and $p_{g}^{* i} \in E_{g}^{*}$ as $i=1, \ldots, n$ satisfying the relationships in (4.1), (5.1), (5.4), and:

$$
\begin{aligned}
& \bar{x}^{i} \text { minimizes }\left\langle\left(p_{\pi}^{*}, p_{g}^{* i}\right), x^{i}\right\rangle \text { over } x^{i} \in \operatorname{cl} P^{i}(\bar{x}), \quad i=1, \ldots, n ; \\
& \bar{y}^{j} \quad \text { maximizes }\left\langle\left(p_{\pi}^{*}, p_{g}^{*}\right), y^{j}\right\rangle \text { over } y^{j} \in \operatorname{cl} S^{j}, \quad j=1, \ldots, m .
\end{aligned}
$$

Proof. Follows from Theorem 5.1 due to the normal cone representation (3.6).

Let us next present a decentralized counterpart of Theorem 5.1 via nonlinear prices.

Theorem 5.5 (decentralized version of the extended second welfare theorem for nonconvex economies with public goods). Let $(\bar{x}, \bar{y})$ be a Pareto (resp. weak Pareto) optimal allocation of the economy $\mathcal{E}$ under the corresponding assumptions of Theorem 5.1 (or the relaxed PSNC assumptions in Remark 5.2), and let $\left(p_{\pi}^{*}, p_{g}^{*}\right) \in E_{\pi}^{*} \times E_{g}^{*}$ and $p_{g}^{* i} \in E_{g}^{*}$ as $i=1, \ldots, n$ be marginal prices satisfying the conditions in (4.1) and (5.1)-(5.4). Then the following assertions hold:

(i) There exist sequences of nonlinear prices $f_{k}=\left(f_{k}^{1}, \ldots, f_{k}^{n}\right)$ and $h_{k}=\left(h_{k}^{1}, \ldots, h_{k}^{m+1}\right)$ as well as sequences of suboptimal allocations

$$
\left(x_{k}, y_{k}\right) \in \prod_{i=1}^{n} \operatorname{cl} P^{i}(\bar{x}) \times \prod_{j=1}^{m} \operatorname{cl} S^{j} \text { with } w_{k}:=\sum_{i=1}^{n} x_{k}^{i}-\sum_{j=1}^{m} y_{k}^{j} \in \operatorname{cl} W
$$

such that for all $k \in \mathbb{N}$ we have the decentralized relationships:

- each $f_{k}^{i}$ and $h_{k}^{j}$ is Fréchet differentiable at $x_{k}^{i}, y_{k}^{j}$, and $w_{k}$ achieving its global minimum over $\operatorname{cl} P^{i}(\vec{x})$ and its global maximum over $\operatorname{cl} S^{j}$ and $\operatorname{cl} W$ at these points for $i=1, \ldots, n$, $j=1, \ldots, m$, and $j=m+1$, respectively;

- $\left(x_{k}, y_{k}, w_{k}\right) \rightarrow(\bar{x}, \dot{\bar{y}}, \bar{w})$ as $k \rightarrow \infty$ with the equilibrium price convergence

$$
\left\{\begin{array}{l}
\left(p_{\pi k}^{*}, p_{g k}^{* i}\right):=\nabla f_{k}^{i}\left(x_{k}^{i}\right) \stackrel{w^{*}}{\rightarrow}\left(p_{\pi}^{*}, p^{* i}\right), \quad i=1, \ldots, n, \\
\left(p_{\pi k}^{* j}, p_{g k}^{* j}\right):=\nabla h_{k}^{j}\left(y_{k}^{j}\right) \stackrel{w^{*}}{\rightarrow}\left(p_{\pi}^{*}, p_{g}^{*}\right), \quad j=1, \ldots, m, \\
\left(p_{\pi k}^{* m+1}, p_{g k}^{* m+1}\right):=\nabla h_{k}^{m+1}\left(w_{k}\right) \stackrel{w^{*}}{\rightarrow}\left(p_{\pi}^{*}, p_{g}^{*}\right) \text { as } k \rightarrow \infty .
\end{array}\right.
$$


(ii) If in addition to the assumptions in (i) the commodity space $E$ is Fréchet smooth, then the nonlinear prices $f_{k}^{i}$ and $h_{k}^{j}$ can be chosen to be Fréchet differentiable on $E$ and such that each $f_{k}^{i}, i=1, \ldots, n$, is convex and achieves its global minimum over $\operatorname{cl} P^{i}(\bar{x})$ uniquely at $x^{i}$ while each $h_{k}^{j}, j=1, \ldots, m+1$, is concave and achieves its global maximum over $\operatorname{cl} S^{j}$ for $j=1, \ldots, m$ and over $\operatorname{cl} W$ for $j=m+1$ uniquely at $y^{j}$ and $w$, respectively.

Proof. It follows by using the arguments similar to those in the proof of Theorem 5.1 with a.pplying there Theorem 4.4 at each approximation step $k \in \mathbb{N}$ and taking into account the strong convergence of the derivatives in (4.20) for $\varepsilon=\varepsilon_{k} \downarrow 0$ as $k \rightarrow \infty$.

We conclude this section with some discussions on possible developments and generalizations of the results obtained for nonconvex economies with public goods.

Remark 5.6 (further developments). Similarly to recent developments for economies with only private goods, we have the following modifications and generalizations of the above versions of the extended second welfare theorem for nonconvex economies with public goods:

(i) Counterparts of the results above hold for strong Pareto optimal allocations and for the new notion of strict Pareto ones [4] under the corresponding modifications of the net demand qualification conditions defined in [4] for economies with only private goods.

(ii) In [16] some refinement of the NDQ condition for Pareto optimal allocation was introduced and employed to the second welfare theorem for economies with only private goods. Analogs of this condition for strong and strict Pareto optimal allocations and the corresponding versions of the second welfare theorem were given in [5] for private goods economies. Following the scheme in [5], we can extend these conditions and results to nonconvex economies with public goods.

\section{Nonconvex Economies with Ordered Commodity Space}

This concluding section of the paper concerns nonconvex economies with public goods and ordered infinite-dimensional spaces of commodities. First we specify the results obtained above for Pareto and weak Pareto optimal allocations and then establish their new counterparts for the case of strong Pareto optimal allocations.

Let $E$ be an ordered Banach space with the closed positive cone

$$
E_{+}:=\{e \in E \mid e \geq 0\}
$$

where the standard partial ordering relation is denoted by ' $\geq$ ' in accordance to the conventional notation in the economic literature. The associated dual closed positive cone $E_{+}^{*}$, which is the closed positive cone of the ordered space $E^{*}$, admits the representation

$$
E_{+}^{*}:=\left\{e^{*} \in E^{*} \mid e^{*} \geq 0\right\}=\left\{e^{*} \in E^{*} \mid\left\langle e^{*}, e\right\rangle \geq 0 \text { for all } e \in E_{+}\right\},
$$

where the order on $E^{*}$ is induced by the given one " $\geq$ " on $E$.

The next theorem provides efficient conditions ensuring the positivity of the marginal prices associated with both private and public goods in our extended second welfare theorem. The result is given for weak Pareto optimal allocations, and hence it holds for any stronger notions of Pareto optimality in the welfare economic model under consideration. 
Theorem 6.1 (price positivity in the extended second welfare theorem for ordered commodities). Let $(\bar{x}, \bar{y})$ be a weak Pareto optimal allocation of the model $\mathcal{E}$ with public goods, where $E=E_{\pi} \times E_{g}$ is an ordered commodity space. Suppose the fulfillment of all the assumptions of Theorem 5.1 but the NDWQ condition and assume instead that each consumer $i \in\{1, \ldots, n\}$ satisfies the following desirability condition:

$$
\operatorname{cl} P^{i}(\bar{x})+E_{+} \subset P^{i}(\bar{x})
$$

Then there exist positive marginal prices $\left(p_{\pi}^{*}, p_{g}^{*}\right) \in E_{\pi}^{*} \times E_{g}^{*}$ and $p_{g}^{* i} \in E_{g}^{*}$ as $i=1, \ldots, n$ for which all the conclusions (4.1), (5.1)-(5.4) hold.

Proof. First observe that the desirability condition (6.1) implies the NDWQ condition by Proposition 2.4(ii). Furthermore, by [31, Lemma 8.12] we have the implication

$$
\left[\Omega-E_{+} \subset \Omega\right] \Longrightarrow\left[N(\bar{e} ; \Omega) \subset E_{+}^{*}\right]
$$

for an arbitrary closed subset $\Omega \subset E$ of an ordered Banach space and any $\bar{e} \in \Omega$. Thus all the conclusions of Theorem 5.1 holds for the weak Pareto optimal allocation $(\bar{x}, \bar{y})$ of the economy $\mathcal{E}$. Then it follows from $(5.2)$ that $p_{\pi}^{*} \geq 0$ and $p_{g}^{* i} \geq 0$ for all $i=1, \ldots, n$. This yields $p_{g}^{*} \geq 0$ by (4.1) and completes the proof of the theorem.

Remark 6.2 (more on price positivity under free disposal or implicit free disposal of commodities). Consider the following conditions of the free disposal type:

- There is $j \in\{1, \ldots, m\}$ such that the $j^{\text {th }}$ firm satisfies the free disposal condition

$$
\operatorname{cl} S^{j}-E_{+} \subset \mathrm{cl} S^{j} .
$$

- The net demand constraint set $W$ exhibits the implicit free disposal of commodities

$$
\operatorname{cl} W-E_{+} \subset \operatorname{cl} W \text {. }
$$

Then it follows from (5.3), (5.4), and (6.2)-(6.4) that we have $\left(p_{\pi}^{*}, p_{g}^{*}\right) \geq 0$ provided that either condition (6.3) holds for some $j \in\{1, \ldots, m\}$, or condition (6.4) is satisfied. In particular (unifying the conclusions of Theorem 6.1 and this remark), for the case of economies with only private goods we have that $p_{\pi}^{*} \geq 0$ if either one of the consumers $i \in\{1, \ldots, n\}$ satisfies the desirability condition (6.1), or one of the firms $j \in\{1, \ldots, m\}$ satisfies the free disposal condition (6.3), or the implicit free disposal condition (6.4) holds.

Next we derive refined versions of the extended second welfare theorem for strong Pareto optimal allocations of economies with public goods. In contrast to the corresponding results of Theorem 5.1 for Pareto and weak Pareto optimal allocations, the NDQ and NDWQ conditions may not be satisfied. Recall that the closed positive cone $E_{+} \subset E$ is generating for $E$ if $E=E_{+}-E_{+}$. The class of normed spaces ordered by their generating positive cones is sufficiently large including, in particular, all Banach lattice (or complete Riesz spaces); see, e.g., $[12,24]$ and the references therein. 
Theorem 6.3 (extended second welfare theorem for strong Pareto optimal allocations). Let $(\bar{x}, \bar{y})$ be a strong Pareto optimal allocation of the economy $\mathcal{E}$ with public goods, and let all the assumptions of Theorem 5.1, except the $N D Q / N D W Q$ conditions, be satisfied. Suppose instead that the positive cone $E_{+}$of the commodity space $E=E_{\pi} \times E_{g}$ is generating, that the sets $S^{j}$ and $W$ are locally closed, around $\bar{y}^{j}$ and $\bar{w}$, respectively, and that one of the following conditions holds:

- the free disposal of commodities

$$
S^{j}-E_{+} \subset S^{j} \text { for some } j \in\{1, \ldots, m\} \text {; }
$$

- the implicit free disposal of commodities (6.4);

- $n>1$, and there is $i_{0} \in\{1, \ldots, n\}$ with $\mathrm{cl} P^{i_{0}}(\bar{x}) \neq \emptyset$ such that

$$
\operatorname{cl} P^{i}(\bar{x})+E_{+} \subset \operatorname{cl} P^{i}(\bar{x}) \text { for some } i \in\{1, \ldots, n\} \backslash\left\{i_{0}\right\} .
$$

Then there exist marginal prices $\left(p_{\pi}^{*}, p_{g}^{*}\right) \in E_{\pi}^{*} \times E_{g}^{*}$ and $p_{g}^{* i} \in E_{g}^{*}$ as $i=1, \ldots, n$ satisfying conditions (4.1) and (5.1)-(5.4). If in addition assumption (6.1) holds for all $i \in\{1, \ldots, n\}$, then we have the price positivity

$$
p^{* i} \geq 0 \text { for all } i=1, \ldots, n \text { and }\left(p_{\pi}^{*}, p_{g}^{*}\right) \geq 0 .
$$

Proof. For definiteness, consider only the case of the implicit free disposal of commodities (6.4); the other two cases of the theorem are treated similarly. Observe first that

$$
\bar{w}=\sum_{i=1}^{n}\left(\bar{x}_{\pi}^{i}, \bar{x}_{g}\right)-\sum_{j=1}^{m} \bar{y}^{j}
$$

is a boundary point of the net demand constraint set $W$. Indeed, assuming the contrary gives us a point $\bar{e}=\left(\bar{e}_{\pi}, \bar{e}_{g}\right) \neq 0$ such that $\bar{w}+\bar{e} \in W$, which implies $(\bar{x}+\bar{e}, \bar{y})$ is a feasible allocation of the economy $\mathcal{E}$. This contradicts the strong Pareto optimality of $(\bar{x}, \bar{y})$. Since $\bar{w}$ is a boundary point of $W$, there is a sequence $\left\{e^{k}\right\} \subset E$ converging to zero and such that

$$
\bar{w}+e^{k} \notin W \text { for all } k \in \mathbb{N} \text {. }
$$

Taking into account that the cone $E_{+}$is generating and employing the the classical KreinSmulian theorem from [1], we find a constant $M>0$ such that for every $e \in E$ there exist vectors $u, v \in E_{+}$satisfying the conditions

$$
e=u-v \text { and } \max \{\|u\|,\|v\|\} \leq M\|e\| .
$$

The latter and the relationship in (6.6) apply the existence of sequences $\left\{u^{k}\right\} \subset E_{+}$and $\left\{v^{k}\right\} \subset E_{+}$with $e^{k}=u^{k}-v^{k}$ and

$$
\bar{w}+u^{k} \notin W \text { for all } k \in \mathbb{N} \text { and } u^{k} \stackrel{E_{+}}{\rightarrow} 0 \text { as } k \rightarrow \infty .
$$

Consider now the sets $\Omega_{1}$ and $\Omega_{2}$ defined in (4.7) and (4.8), respectively, where the closure operation for $S^{j}$ and $W$ can be omitted. Let us show that $(\bar{x}, \bar{y}, \bar{w}) \in \Omega_{1} \cap \Omega_{2}$ is an extremal point of the system $\left\{\Omega_{1}, \Omega_{2}\right\}$. We need to find a sequence $\left\{a^{k}\right\} \subset E^{n+m+1}$ with 
$a^{k} \rightarrow 0$ as $k \rightarrow \infty$ such that the extremality condition (4.9) is satisfied. To proceed, define $a^{k}:=\left(0, \ldots, u^{k}\right)$ and suppose that $(4.9)$ does not hold along this sequence $\left\{a^{k}\right\}$, i.e., there are $\left(x^{k}, y^{k}, w^{k}\right) \in \Omega_{1}$ such that $\left(x^{k}, y^{k}, w^{k}-u^{k}\right) \in \Omega_{2}$ for all $k \in \mathbb{N}$. This yields that

$$
\sum_{i=1}^{n}\left(x_{\pi}^{k i}, x_{g}^{k}\right)-\sum_{j=1}^{m} y^{k j}=w^{k}-u^{k} \in W
$$

which implies that $\left(x^{k}, y^{k}\right)$ is a feasible allocation of the economy $\mathcal{E}$. By the strong Pareto optimality of $(\bar{x}, \bar{y})$ we get that $\left(x^{k}, y^{k}\right)=(\bar{x}, \bar{y})$ for all $k \in \mathbb{N}$ and hence

$$
\begin{aligned}
\bar{w}+u^{k} & = & & \sum_{i=1}^{n}\left(\bar{x}_{\pi}^{i}, \bar{x}_{g}\right)-\sum_{j=1}^{m} \bar{y}^{j}+u^{k} \\
& = & & \sum_{i=1}^{n}\left(x_{\pi}^{k i}, x_{g}^{k}\right)-\sum_{j=1}^{m} y^{k j}+u^{k} \\
& = & & \left(w^{k}-u^{k}\right)+u^{k}=w^{k} \in W, \quad k \in \mathbb{N},
\end{aligned}
$$

which contradicts (6.7) and thus justifies the extremality of $(\bar{x}, \bar{y}, \bar{w})$. Applying now the extremal principle from Theorem $3.7(\mathrm{i})$ to the system $\left\{\Omega_{1}, \Omega_{2}\right\}$ at $(\bar{x}, \bar{y}, \bar{w})$ and arguing as in the proof of Theorem 4.1; we obtain approximate marginal prices satisfying all the conclusions of the latter theorem with $\varepsilon=\varepsilon_{k} \downarrow 0$ as $k \rightarrow \infty$. Passing then to the limit as $k \rightarrow \infty$ as in the prove of Theorem 5.1 under the SNC assumptions made allows us to arrive at the marginal prices $\left(p_{\pi}^{*}, p_{g}^{*}, p_{g}^{* i}\right)$ satisfying conditions (4.1) and (5.1)-(5.4). Finally, the price positivity (6.5) under the additional assumption (6.1) follows from Theorem 6.1.

\section{References}

[1] Abramovich YA, Aliprantis CD (2002) An invitation to operator theory. American Mathematical Society, Providence, RI

[2] Alpirantis CD, Tourky R, Yannelis NC (2001) A theory of values with nonlinear prices. Equilibrium analysis beyond vector lattices. J Econ Theory 100:22-72

[3] Arrow KJ (1951) An extension of the basic theorem of classical welfare economics. In: Neyman J (ed) Proceedings of the Second Berkeley Symposium on Mathematical Statistics and Probability, pp 507-532. University of California, Berkeley, CA

[4] Bao TQ, Mordukhovich BS (2010) Set-valued optimization in welfare economics. Adv Math Econ 13:113-153

[5] Bao TQ, Mordukhovich BS (2010) Refined necessary conditions in multiobjective optimization with applications to microeconomic modeling. Comm Pure Appl Anal (to appear)

[6] Bonnisseau JM, Cornet B (1988) Valuation equilibrium and Pareto optimum, J Math Econ 17:293-308 
[7] Bonnisseau JM, Lachiri $O$ (2006) About the second theorem of welfare economics with stock markets. Pacif J Math 2:469-485

[8] Borwein JM, Strójwas HM (1985) Tangential approximations. Nonlinear Anal 9:13471366

[9] Cornet B (1986) The second welfare theorem in nonconvex economics. CORE discussion paper, no 8630

[10] Debreu G (1951) The coefficient of resource utilization. Econometrica 19:273-292

[11] Debreu G, Hilderbrand W (1968) Existence of equilbria in market with infinitedimensional commodity space. University of California Berkeley, CA (Technical report)

[12] Florenzano M, Gourdel P, Jofré A (2006) Supporting weakly Pareto optimal allocations in infinite-dimensional nonconvex economies. J Econ Theory 29:549-564

[13] Foley DK (1967) Lindal's solution and the core of an economic with public goods. Econometrica 38:66-72

[14] Guesnerie R (1975) Pareto optimality in nonconvex economies. Econometrica 43:1-29

[15] Hicks JR (1939) The foundations of welfare economics. Econ J 49:696-712

[16] Ioffe $\mathrm{AD}$ (2009) Variational analysis and mathematical economics, I: subdifferential calculus and the second theorem of welfare economics. Adv Math Econ 12:71-95

[17] Jofré A (2000) A second welfare theorem in nonconvex economies. In: Théra (ed), Constructive, experimental and nonlinear analysis. CMS Conference Proceedings, vol 27, pp 123-164

[18] Jofré A, Rivera Cayopi J (2006) A nonconvex separation property and some applications. Math Program 108:37-51

[19] Khan MA (1991) Ioffe's normal cone and the foundation of welfare economics: the infinite dimensional theory. J Math Anal Appl 161:284-298

[20] Khan MA (1999) The Mordukhovich normal cone and the foundations of welfare economics. J Public Econ Theory 1:309-338

[21] Khan MA, Vohra R (1987) An extension of the second welfare theorem to economies with nonconvexity and public goods. Q J Econ 102:223-245

[22] Lang O (1942) The foundation of welfare economics. Econometrica 10:215-228

[23] Malcolm GG, Mordukhovich BS (2001) Pareto optimality in nonconvex economies with infinite-dimensional commodity spaces. Journal Global Optim 20:323-346

[24] Mas-Colell A (1986) The price equilibrium existence problem in topological vector lattices. Econometrica 54:1039-1053 
[25] Milleron JC (1972) Theory of value with public goods: A survey article. J Econ Theory $5: 419-477$

[26] Mordukhovich BS (1976) Maximum principle in time optimal control problems with nonsmooth constraints. J Appl Math Mechanics 40:960-969

[27] Mordukhovich BS (1980) Metric approximations and necessary optimality conditions for general classes of nonsmooth extremal problems. Soviet Math Dokl 22: 526-530

[28] Mordukhovich BS (2000) Abstract extremal principle with applications to welfare economics. J Math Anal Appl 251: 187-216

[29] Mordukhovich BS (2005) Nonlinear prices in nonconvex economies with classi cal Pareto and strong Pareto optimal allocations. Positivity 9:541-568

[30] B. S. Mordukhovich (2006) Variational analysis and generalized differentiation, I: basic theory. Grundlehren Series (Fundamental Principles of Mathematical Sciences), vol 330. Springer, Berlin

[31] Mordukhovich BS (2006) Variational analysis and generalized differentiation, II: applications. Grundlehren Series (Fundamental Principles of Mathematical Sciences), vol 331. Springer, Berlin

[32] Rockafellar RT (1979) Directional Lipschitzian functions and subdifferential calculus. Proc London Math Soc 39:331-355

[33] Samuelson PA (1943) Further commentary on welfare economics. Amer Econ Rev $33: 605-607$

[34] Samuelson PA (1947) Foundation of economic analysis. Harvard University Press, Cambridge, $M A$

[35] Samuelson PA (1954) The pure theory of public expenditure. Rev Econ Stat 36:387-389

[36] Simone AD and Graziano MG (2004) The pure theory of public goods: the case of many commodities. J Math Econ 40:847-868

[37] Villar A (2000) Equilibrium and efficiency in production economies. Springer, Berlin 